\title{
Optimal energy conversion through antiadiabatic driving breaking time-reversal symmetry
}

\author{
L. M. Cangemi $\odot,{ }^{1,2, *}$ M. Carrega, ${ }^{3}$ A. De Candia, ${ }^{1,2,4}$ V. Cataudella, ${ }^{1,2,4}$ G. De Filippis, ${ }^{1,2,4}$ \\ M. Sassetti, ${ }^{5,3}$ and G. Benenti $\odot^{6,7,8}$ \\ ${ }^{1}$ Dipartimento di Fisica “E. Pancini,” Università di Napoli “Federico II,” Complesso di Monte S. Angelo, via Cinthia, 80126 Napoli, Italy \\ ${ }^{2}$ CNR/SPIN, clo Dipartimento di Fisica "E. Pancini”, Complesso di Monte S. Angelo, via Cinthia-80126-Napoli, Italy \\ ${ }^{3}$ CNR-SPIN, Via Dodecaneso 33, 16146 Genova, Italy \\ ${ }^{4}$ INFN, Sezione di Napoli, Complesso Universitario di Monte S. Angelo, I-80126 Napoli, Italy \\ ${ }^{5}$ Dipartimento di Fisica, Università di Genova, Via Dodecaneso 33, 16146 Genova, Italy \\ ${ }^{6}$ Center for Nonlinear and Complex Systems, Dipartimento di Scienza e Alta Tecnologia, \\ Università degli Studi dell'Insubria, via Valleggio 11, 22100 Como, Italy \\ ${ }^{7}$ Istituto Nazionale di Fisica Nucleare, Sezione di Milano, via Celoria 16, 20133 Milano, Italy \\ ${ }^{8}$ NEST, Istituto Nanoscienze-CNR, I-56126 Pisa, Italy
}

(Received 22 September 2020; accepted 15 February 2021; published 12 March 2021)

\begin{abstract}
Starting with the Carnot engine, the ideal efficiency of a heat engine has been associated with quasistatic transformations and vanishingly small output power. Here, we exactly calculate the thermodynamic properties of an isothermal heat engine, in which the working medium is a periodically driven underdamped harmonic oscillator, focusing instead on the opposite, antiadiabatic limit, where the period of a cycle is much shorter than the system's timescales. We show that in that limit it is possible to approach the ideal energy conversion efficiency $\eta=1$, with finite output power and vanishingly small relative power fluctuations. The simultaneous realization of all the three desiderata of a heat engine is possible thanks to the breaking of time-reversal symmetry. We also show that non-Markovian dynamics can further improve the power-efficiency trade-off.
\end{abstract}

DOI: 10.1103/PhysRevResearch.3.013237

\section{INTRODUCTION}

Since its inception, the development of thermodynamics and its technological applications have been boosted by fundamental questions [1-8]. A key question is what are the ultimate bounds to the performance of heat engines [9-20]. It is desirable that a heat engine operates close to the ideal efficiency (the Carnot efficiency when the working medium exchanges heat with reservoirs at different temperatures, or unit efficiency for an isothermal engine), delivers large power, and exhibits small power fluctuations [21,22]. Physical intuition tells us that the ideal efficiency can be obtained only if the energy conversion process is reversible. As a thermodynamic reversible transformation is quasistatic, the thermodynamic cycle takes an infinite time, and therefore the output power vanishes. On the other hand, the second law of thermodynamics by itself does not forbid the possibility of achieving the ideal efficiency at finite power [23]. Several studies have shown that it is possible to come arbitrarily close to the ideal efficiency [24-31], with finite power. However, diverging power fluctuations, as in the case when the working

\footnotetext{
*lorismaria.cangemi@unina.it

Published by the American Physical Society under the terms of the Creative Commons Attribution 4.0 International license. Further distribution of this work must maintain attribution to the author(s) and the published article's title, journal citation, and DOI.
}

medium is at the verge of a phase transition [26], or the necessity of precisely engineering the scaling of model parameters [30,31], make such engines impractical [21,30,32].

Thermodynamic uncertainty relations (TURs) [33-48] set a lower bound on the time-integrated relative fluctuation of an arbitrary current, which diverges when the dissipationless limit required for ideal efficiency is achieved. The application of such relations to the "work current" (i.e., the output power) leads, for steady-state heat engines with time-reversal symmetry (TRS), to a trade-off between efficiency, power, and fluctuations [22]. Consequently, approaching the ideal efficiency with finite power implies diverging fluctuations. On the other hand, such result does not apply for cyclic heat engines, for which a less restrictive trade-off has been derived [46] for overdamped Markovian dynamics. This interesting result raises the following questions: (i) is it possible to approach the ideal efficiency at finite power and finite (or even vanishing) relative power fluctuations in a purely dynamical model, without using the overdamped and Markov or other master equation approximations? (ii) Counterintuitive as it may be, is it possible to obtain such result far from the quasistatic limit? Possibly in the complementary, antiadiabatic limit, where the period of a cycle is much shorter than the system's timescales?

To address these questions, we consider a paradigmatic model where the working medium is a harmonic oscillator coupled to a thermal bath. The oscillator's canonically conjugated variables are separately coupled to periodic drives. This system can act as an isothermal heat engine. This model has several advantages: (i) it can be exactly solved, also in the 
far-from-equilibrium regime and for strong system-bath coupling, without resorting to the overdamped or other approximations; (ii) it is possible to break time reversibility and to address all driving regimes, from the quasistatic to the antiadiabatic one; and (iii) non-Markovian effects are naturally included and can be tuned by engineering the bath spectral density. Here, we show that the ideal, unit efficiency for energy conversion is achieved both in the quasistatic and in the antiadiabatic limits. While in the first case the output power vanishes, in the latter we also obtain the other two desiderata of a heat engine, that is, finite power and vanishing relative power fluctuations. Non-Markovian effects can then further improve the power-efficiency trade-off while approaching the unit-efficiency limit. Finally, we clarify the necessity of breaking TRS to obtain the above results.

The paper is organized as follows. In Sec. II, we present the model under investigation and its exact solution. In Sec. III, we discuss its performance as an isothermal engine, while in Sec. IV, we present our main findings, focusing in particular on the fast driving regime. Section V contains our main conclusions. Technical details are provided in some appendices.

\section{DRIVEN HARMONIC ISOTHERMAL HEAT ENGINE}

We consider energy conversion process through a driven (quantum) resonator connected to a thermal reservoir, whose total Hamiltonian is $H(t)=H_{\mathrm{S}}(t)+H_{\mathrm{R}}+H_{\mathrm{SR}}$. As working medium we consider a single harmonic oscillator, linearly coupled through its canonical degrees of freedom to timeperiodic external fields driving it out-of equilibrium,

$$
H_{\mathrm{S}}(t)=\frac{p^{2}}{2 m}+\frac{1}{2} m \omega_{0}^{2} x^{2}-\varepsilon_{1}(t) x-\varepsilon_{2}(t) p,
$$

where $m$ and $\omega_{0}$ are the mass and characteristic frequency of the oscillator, respectively (hereafter we set $\hbar=k_{\mathrm{B}}=1$ ). External drives are periodic functions, $\varepsilon_{i}(t)=\varepsilon_{i}(t+\mathcal{T})(i=$ $1,2)$, with period $\mathcal{T}=2 \pi / \omega$.

The system is unavoidably coupled with its surrounding environment, which behaves as a thermal bath causing noise and dissipation. This can be described by the standard Caldeira-Leggett model $[49,50]$, in terms of an infinite set of harmonic oscillators,

$$
H_{\mathrm{R}}+H_{\mathrm{SR}}=\sum_{k=1}^{\infty}\left[\frac{P_{k}^{2}}{2 m_{k}}+\frac{m_{k} \omega_{k}^{2}}{2}\left(X_{k}-\frac{c_{k}}{m_{k} \omega_{k}^{2}} x\right)^{2}\right],
$$

linearly coupled to the oscillator position $x$. The system can act as an isothermal heat engine [51-54]. Physically, as sketched in Fig. 1, when a given amount of work is put in an input channel, the system delivers part of it in output, while dissipating a given amount into the thermal reservoir.

The system described by Eq. (1) can model a wide range of experimental realizations, covering both classical and quantum regimes. In the former case, one can consider an electronic RLC circuit coupled to both external timedependent electric and magnetic fields. An ac voltage couples with the position variable (the charge), while the magnetic field via electromagnetic induction couples with the current. One can also think about a driven quantum LC circuit [55-58] implemented by superconducting circuit elements [59]. The

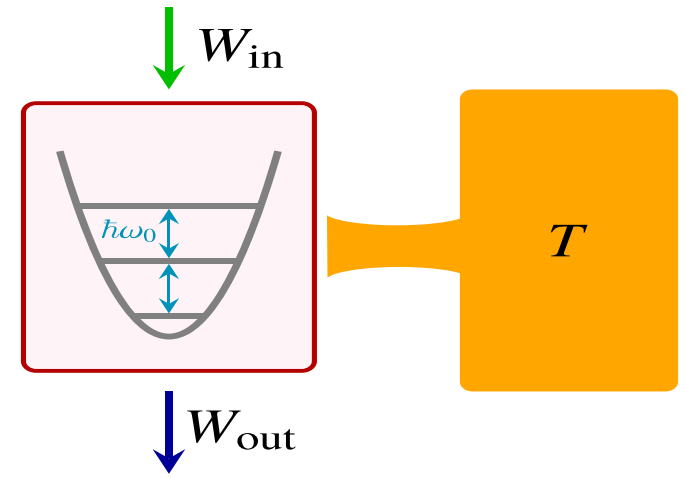

FIG. 1. Sketch of the driven harmonic isothermal heat engine. A quantum resonator is periodically driven along two different channels. It is connected to a thermal bath at temperature $T$. An amount of work per cycle is done on the system along a given channel $\left(W_{\text {in }}\right)$, it is partially converted in output along the other $\left(W_{\text {out }}\right)$ and partially dissipated into the bath.

conjugate variables are the flux and charge variables $(\varphi, Q)$, the capacitance $C$ plays the role of the mass $m$ and the oscillator frequency $\omega_{0}=1 / \sqrt{L C}$, with $L$ the circuit inductance. Here, one driving can be engineered, for example, by means of a capacitive coupling of an ac-voltage to the charge of the circuit [57]. The other driving field can be obtained by using other inductive elements, which couple to the circuit via mutual inductance. The bath in Eq. (2) phenomenologically accounts for dissipative effects on the LC circuit arising from the external circuitry impedance [56].

The equations of motion (EOM) read

$$
\begin{aligned}
\langle\dot{x}(t)\rangle= & \frac{\langle p(t)\rangle}{m}-\varepsilon_{2}(t), \\
& \langle\ddot{x}(t)\rangle+\int_{-\infty}^{t} d t^{\prime} \gamma\left(t-t^{\prime}\right)\left\langle\dot{x}\left(t^{\prime}\right)\right\rangle+\omega_{0}^{2}\langle x(t)\rangle \\
= & \frac{\varepsilon_{1}(t)}{m}-\dot{\varepsilon}_{2}(t),
\end{aligned}
$$

with $\langle A\rangle \equiv \operatorname{Tr}\left\{\rho_{\text {tot }}\left(t_{0}\right) A\right\}$ representing the quantum average of a generic operator $A$ with factorized initial preparation (at time $\left.t_{0}=-\infty\right) \rho_{\text {tot }}\left(t_{0}\right)=\rho_{S}\left(t_{0}\right) \otimes \rho_{R}\left(t_{0}\right)$ given by the product between the initial system density $\rho_{S}\left(t_{0}\right)$ and the density matrix of the bath alone, which is assumed in thermal equilibrium $\rho_{R}\left(t_{0}\right)=\exp \left(-H_{\mathrm{R}} / T\right) / \operatorname{Tr}\left\{\exp \left(-H_{\mathrm{R}} / T\right)\right\}$. Notice that Eq. (3), which is valid both in the classical and in the quantum case, can be derived from an underdamped Langevin equation in the presence of time-dependent forces (see Appendix A). The memory kernel $\gamma(t)$ describes friction and can be linked to the bath spectral density $J(\omega)$ as $\gamma(t)=$ $\frac{2}{\pi m} \theta(t) \int_{0}^{+\infty} d \omega J(\omega) \cos (\omega t) / \omega$ (see Appendix A). In the continuum limit, and assuming a Lorentzian high-frequency bath cutoff $\omega_{\mathrm{c}}, J(\omega)$ reads

$$
J(\omega)=m \gamma_{s} \bar{\omega}^{1-s} \frac{\omega^{s}}{1+\left(\omega / \omega_{\mathrm{c}}\right)^{2}} .
$$

Here, $\gamma_{s}$ is the friction amplitude, $\bar{\omega}$ the characteristic bath frequency and the index $s$ distinguishes among different kinds of dissipation: Ohmic $(s=1)$, sub-Ohmic $(s<1)$ and 
super-Ohmic $(s>1)$ [50]. For $s \neq 1$, the system's dynamics is non-Markovian in the whole range of frequency.

The linearity of the EOM guarantees that the response to the external fields $\varepsilon_{i}(t)$ can be computed exactly at any field strength. The expectation values of $\mathbf{z}(t) \equiv(x(t), p(t))$ can be written in terms of a generalized susceptibility matrix $\chi_{i j}(t-$ $\left.t^{\prime}\right)=-i \theta\left(t-t^{\prime}\right)\left\langle\left[z_{i}(t), z_{j}\left(t^{\prime}\right)\right]\right\rangle_{T}[60]$ as

$$
\left\langle z_{i}(t)\right\rangle=-\sum_{j=1,2} \int_{-\infty}^{+\infty} d t^{\prime} \chi_{i j}\left(t-t^{\prime}\right) \varepsilon_{j}\left(t^{\prime}\right) .
$$

Solving Eq. (3) in Fourier space, the analytic expressions for $\chi_{i j}(\omega)=\int_{-\infty}^{+\infty} d \tau \chi_{i j}(\tau) e^{i \omega \tau}$ follow :

$$
\begin{aligned}
& \chi_{11}(\omega)=\frac{1}{m} \frac{1}{\omega^{2}-\omega_{0}^{2}+i \omega \gamma(\omega)}, \\
& \chi_{22}(\omega)=-m+m^{2} \omega^{2} \chi_{11}(\omega), \\
& \chi_{12}(\omega)=-\chi_{21}(\omega)=i m \omega \chi_{11}(\omega),
\end{aligned}
$$

where $\gamma(\omega)=\int_{0}^{+\infty} \gamma(\tau) e^{i \omega \tau} d \tau$ is the frequency dependent damping (see Appendix A). It is worth to note that linearity imposes that $\chi_{i j}(\omega)$ do not depend on temperature.

\section{ENGINE PERFORMANCE}

In the periodic steady state sustained by the drives, the total work per cycle can be defined as [61] $W=$ $\int_{0}^{\mathcal{T}} d t \operatorname{Tr}\left\{\frac{\partial H(t)}{\partial t} \rho_{\text {tot }}(t)\right\}$, with $\rho_{\text {tot }}(t)$ the total density matrix at time $t$. The work contributions associated to the drives are identified as

$$
W_{i}=-\int_{0}^{\mathcal{T}} d t \dot{\epsilon}_{i}(t)\left\langle z_{i}(t)\right\rangle,
$$

with mean power per cycle $P_{i}=W_{i} / \mathcal{T}$. We consider the parameter regions where energy is exchanged between an input channel with $P_{\text {in }}=P_{2}>0$ and an output one with $P_{\text {out }}=-P_{1}$ $\left(P_{1}<0\right)$ [62], i.e., a given amount of work per cycle is converted from $W_{\text {in }}=W_{2}$ to $W_{\text {out }}=-W_{1}$ with efficiency

$$
\eta \equiv \frac{W_{\text {out }}}{W_{\text {in }}}=\frac{P_{\text {out }}}{P_{\text {in }}} .
$$

For sake of definiteness, the external drives are chosen as $\varepsilon_{1}(t)=\varepsilon_{1} \sin \omega t, \varepsilon_{2}(t)=\varepsilon_{2} \cos (\omega t-\varphi)$, with $\varphi \in[0,2 \pi)$ a possible phase shift. Exact espressions for the mean powers along the two channels can be computed starting from Eq. (3), and can be compactly written as

$$
P_{i}=\varepsilon_{i} \sum_{j=1,2} \varepsilon_{j} \mathcal{L}_{i j},
$$

where $\mathcal{L}_{i j}$ represent the elements of the generalized Onsager matrix of the isothermal heat engine, which exactly describes the response of the working medium to the external fields. In terms of susceptibilities $\chi_{i j}$, they read

$$
\begin{aligned}
\mathcal{L}_{11}(\omega) & =-\frac{\omega}{2} \Im\left[\chi_{11}(\omega)\right], \quad \mathcal{L}_{22}(\omega)=-\frac{m^{2} \omega^{3}}{2} \Im\left[\chi_{11}(\omega)\right], \\
\mathcal{L}_{12}(\omega, \varphi) & =-\frac{m \omega^{2}}{2}\left(\sin \varphi \Re\left[\chi_{11}(\omega)\right]+\cos \varphi \Im\left[\chi_{11}(\omega)\right]\right), \\
\mathcal{L}_{21}(\omega, \varphi) & =\mathcal{L}_{12}(\omega,-\varphi),
\end{aligned}
$$

We note that, due to the linearity of the model, they are temperature independent. It is worth also to underline that the phase difference $\varphi$ controls the asymmetry of the Onsager matrix: for $\varphi \neq 0, \pi$, TRS is broken, with the device operating at maximum asymmetry, $\mathcal{L}_{12}=-\mathcal{L}_{21}$, for, e.g., $\varphi=3 \pi / 2$.

Fluctuations can be evaluated by computing the variance $D_{i}$ of $P_{i}$. Via fluctuation-dissipation theorem the time-averaged variance can be written as $D_{i}=$ $\varepsilon_{i}^{2} \omega \operatorname{coth}[\omega /(2 T)] \mathcal{L}_{i i}$ (see Appendix B), also valid for any field strength. Contrary to mean powers and efficiency, which depend only on $\mathcal{L}_{i j}$, quantum fluctuations explicitly depend on temperature, reaching only at high temperature their classical expression $\propto T$ [50].

\section{RESULTS}

We now characterize energy conversion performance, focusing on the regimes where the efficiency $\eta$ is close to the ideal value $\eta=1$. To this end, for any given value of $\omega$ and $\varphi$, we consider the maximum efficiency $\eta_{\mathrm{ME}}$, obtained by maximizing $\eta$ over the output amplitude $\varepsilon_{1}$, for a fixed $\varepsilon_{2}$. All results discussed below are obtained considering the highfrequency limit for the bath cutoff $\omega_{\mathrm{c}} \gg \omega, \omega_{0}, \bar{\omega}, \gamma_{s}$. We stress that it is indeed in this regime that the Caldeira-Leggett model describes a well behaved thermal bath. Hereafter, we focus on the case with broken TRS, showing that it is possible to achieve finite output power $P_{\mathrm{out}, \mathrm{ME}}>0$ and vanishing relative output power fluctuations $\Sigma_{\mathrm{ME}}=\sqrt{D_{\mathrm{out}, \mathrm{ME}} / P_{\mathrm{out}, \mathrm{ME}}^{2}}$ with $\eta_{\mathrm{ME}} \rightarrow 1$. Explicit results are reported for the maximally asymmetric case $\varphi=3 \pi / 2$; for any value of $\varphi$ breaking TRS, $\mathcal{L}_{12}=-\mathcal{L}_{21}$ is anyway recovered in the antiadiabatic limit (see Appendix B).

In Fig. 2, we show the efficiency $\eta_{\mathrm{ME}}$, the input and output power behaviors, and the relative power fluctuations at $\mathrm{ME}$ as a function of driving frequency $\omega$, in the case of Ohmic damping $(s=1)$. As it is clear from Fig. 2(a), the ideal limit $\eta_{\mathrm{ME}}=1$ is approached in two opposite regimes. However, looking at Fig. 2(b), the output power $P_{\text {out, } \mathrm{ME}}$ tends to vanish at small frequency $\omega \rightarrow 0$ (quasistatic limit). On the contrary, it takes finite value in the antiadiabatic driving regime $\omega_{0}, \bar{\omega}, \gamma_{s} \ll \omega \ll \omega_{\mathrm{c}}$. More precisely, in this regime the output power has a linear scaling with frequency, $P_{\mathrm{out}, \mathrm{ME}} \approx$ $\frac{m \varepsilon_{2}^{2}}{2}\left(\omega-2 \gamma_{s}(\omega / \bar{\omega})^{s-1}\right)$. The energy conversion is mediated by the bath, with the mean dissipated power $P_{\text {in, }, \mathrm{EE}}-P_{\text {out, } \mathrm{ME}}=$ $\left\langle\dot{H}_{\mathrm{R}}\right\rangle \approx m \varepsilon_{2}^{2} \gamma_{s}(\omega / \bar{\omega})^{s-1}$, where also $\left\langle\dot{H}_{\mathrm{R}}\right\rangle$ is averaged over one driving period. In the antiadiabatic regime, the efficiency approaches the ideal value as $\eta_{\mathrm{ME}} \rightarrow 1-2\left(\gamma_{\mathrm{s}} / \bar{\omega}\right)(\omega / \bar{\omega})^{s-2}$, with $0<s<2$ (see Appendixes C and D).

Breaking of TRS (see discussion below) and antiadiabatic driving allow us to approach the ideal efficiency with finite value of output power without affecting the engine precision. Indeed, as shown in Fig. 2(c), the relative fluctuations $\Sigma_{\mathrm{ME}}$ are suppressed by increasing the frequency. The asymptotic scaling of $\Sigma_{\mathrm{ME}}$, for generic dissipation, is given by $A\left\{\operatorname{coth}[\omega /(2 T)](\omega / \bar{\omega})^{(s-2)}\right\}^{1 / 2}$, with the prefactor $A=$ $\left(2 \gamma_{s} / m \bar{\omega} \varepsilon_{2}^{2}\right)^{1 / 2}$ (see Appendix D), confirming the decrease of $\Sigma_{\mathrm{ME}}$ with the increase of $\omega$, for $0<s<2$. In passing, we note that $\Sigma_{\mathrm{ME}}$ depends on temperature, implying lower fluctuations at low temperatures (quantum regime). 

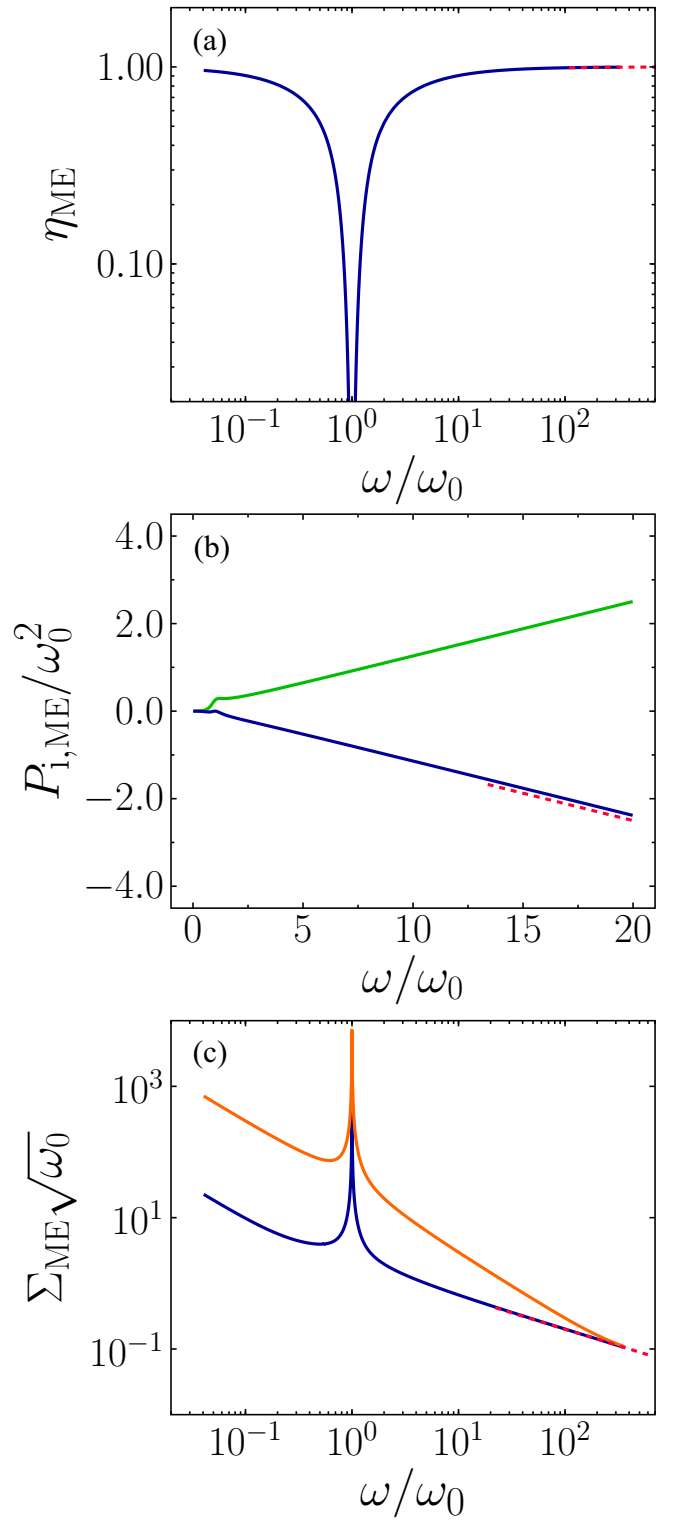

FIG. 2. Isothermal engine performance at ME as a function of the driving frequency $\omega$, for Ohmic friction $s=1$. (a) shows the efficiency $\eta_{\mathrm{ME}}$, (b) the input (green solid line) and output (blue solid line) powers, and (c) the relative fluctuations at low temperature $T=$ $0.1 \omega_{0}$ (blue solid line) and high temperature $T=100 \omega_{0}$ (orange solid line). Dashed lines correspond to the asymptotic power-law behavior for $\omega \gg \omega_{0}, \gamma_{1}$. All quantities are scaled in units of $\omega_{0}$. Other parameters are $m \varepsilon_{2}^{2}=0.25 \omega_{0}, \gamma_{1}=0.5 \omega_{0}, \bar{\omega}=\omega_{0}$ and $\omega_{c}=$ $4 \times 10^{4} \omega_{0}$

To quantify engine performance, it is interesting to look at the scaling property of the output power while approaching the ideal limit $\eta_{\mathrm{ME}} \rightarrow 1$, in the antiadiabatic regime (see Appendix D). This is depicted in Fig. 3. Here, $P_{\text {out, } \mathrm{ME}}$ versus $1-\eta_{\mathrm{ME}}$ shows different power-law behaviors for different values of $s$, at fixed damping strength. Non-Ohmic environment, implying memory effects, qualitatively changes the scaling of power versus efficiency: $P_{\text {out }, \mathrm{ME}} \propto\left(1-\eta_{\mathrm{ME}}\right)^{1 /(s-2)}$. Since the output power $P_{\text {out,ME }} \propto \omega$ does not depend on $s$ at leading order, it follows that sub-Ohmic dissipation $(s<1)$ allows the

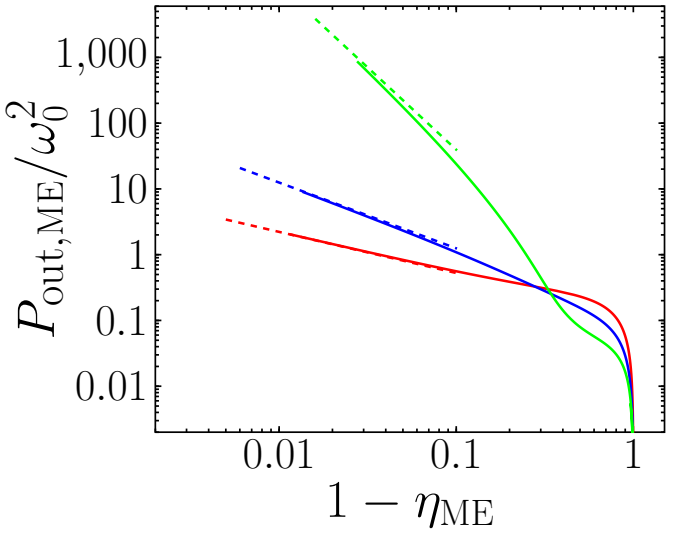

FIG. 3. Output power plotted vs $1-\eta_{\mathrm{ME}}$ at ME in the antiadiabatic regime, for $s=0.4$ (red line), 1 (blue line), and 1.6 (green line). The dashed lines correspond to the scaling $P_{\mathrm{out}, \mathrm{ME}} \propto\left(1-\eta_{\mathrm{ME}}\right)^{1 /(s-2)}$. Other parameter values are as in Fig. 2.

conversion efficiency $\eta_{\mathrm{ME}}$ to get closer to the unit-efficiency limit, with respect to the other cases. Note that the bath properties drastically affect the entropy production rate $\sigma=\left(P_{\text {in }}-\right.$ $\left.P_{\text {out }}\right) / T$. Since $\sigma_{\mathrm{ME}} \propto \gamma_{s} \omega^{s-1}$ for large $\omega$ but always keeping $\omega \ll \omega_{\mathrm{c}}$, vanishing entropy production rate is achieved in the sub-Ohmic regime.

We mention that our model violates in a broad range of parameter values the TUR bound [46], derived for periodically driven, overdamped Markovian systems (see Appendix E for a detailed discussion). In particular, while for our model in the antiadiabatic regime such bound predicts $\mathcal{Q}_{\mathrm{ME}} \equiv \sigma_{\mathrm{ME}} \Sigma_{\mathrm{ME}}^{2} \geqslant 2$, we obtain $\mathcal{Q}_{\mathrm{ME}} \rightarrow 0$ at large frequency (see Appendix E).

Finally, we comment on the role played by TRS breaking. Provided that linear response holds, a general trade-off between efficiency, power, and relative fluctuations can be derived following Ref. [63]. For an isothermal heat engine it is

$$
\mathcal{Q}_{\mathrm{ME}}=\left(\frac{1}{\eta_{\mathrm{ME}}}-1\right) \frac{P_{\mathrm{out}, \mathrm{ME}}}{T} \Sigma_{\mathrm{ME}}^{2} \geqslant \mathcal{B} \equiv \frac{2}{1+S_{L}^{2}},
$$

where $S_{L}$ is related to the asymmetry of the Onsager matrix [63]. In particular, for systems with TRS $S_{L}=0$ and one recovers the bound of Ref. [22], which forbids ideal efficiency at finite power, unless fluctuations diverge. Our model, although not restricted to small values of the external driving strength, is written in terms of generalized Onsager coefficients and therefore satisfies the bound (11) (see Appendix E). Breaking TRS implies a diverging asymmetry coefficient $S_{L}$ in the antiadiabatic regime, i.e., $\mathcal{B} \rightarrow 0$. Therefore, in this limit, the trade-off parameter $\mathcal{Q}_{\mathrm{ME}} \geqslant 0$, that is, it becomes irrelevant for the engine performance.

\section{CONCLUSIONS}

By modeling an isothermal heat engine as a driven harmonic oscillator coupled to a thermal bath, we showed that in the antiadiabatic regime it is possible to achieve the ideal energy conversion, with simultaneous finite, and precise, output power. Essential ingredients for our results are the breaking of TRS and the fact that the exact underdamped dynamics of the 
working medium is considered. We stress that in the opposite, quasistatic limit the above desirable features cannot be jointly observed, since the output power vanishes when the ideal, unit efficiency is approached.

It would be interesting to investigate the exact underdamped dynamics in the case of a harmonic oscillator coupled to two heat baths at different temperatures [64-69]. In particular, to verify if also in this case it is possible to approach in the antiadiabatic regime the ideal, Carnot limit at finite power and with finite or even vanishing relative fluctuations. Further natural generalizations of our model could be obtained by considering engines with a more complex working medium, like coupled oscillators or qubit-cavity systems.

\section{ACKNOWLEDGMENTS}

We acknowledge G. Strini, F. Giazotto, S. Gasparinetti for useful discussions. A.d.C. acknowledges financial support of the MIUR PRIN 2017WZFTZP "Stochastic forecasting in complex systems."

\section{APPENDIX A: SOLUTION OF THE EQUATION OF MOTION}

We start from the Hamiltonian reported in the main text (recall that we set $\hbar=1$ and $k_{B}=1$ ),

$$
H=H_{\mathrm{S}}(t)+H_{\mathrm{R}}+H_{\mathrm{SR}},
$$

where

$$
H_{\mathrm{S}}(t)=\frac{p^{2}}{2 m}+\frac{1}{2} m \omega_{0}^{2} x^{2}-\varepsilon_{1}(t) x-\varepsilon_{2}(t) p
$$

is the driven resonator Hamiltonian,

$$
H_{\mathrm{R}}=\sum_{k=1}^{\infty}\left[\frac{P_{k}^{2}}{2 m_{k}}+\frac{m_{k} \omega_{k}^{2} X_{k}^{2}}{2}\right]
$$

is the collection of infinite harmonic oscillators describing the reservoir and

$$
H_{\mathrm{SR}}=-x \sum_{k=1}^{\infty} c_{k} X_{k}+x^{2} \sum_{k=1}^{\infty} \frac{c_{k}^{2}}{2 m_{k} \omega_{k}^{2}},
$$

represents the coupling, bilinear in the position operators of system and reservoir. Notice that the last term in Eq.(A4) contains only an operator acting in the system Hilbert space but it depends on the coupling constants $c_{k}$. The physical reason for the inclusion of this term is to avoid the potential renormalization introduced by the first term in Eq.(A4) [50].

The driven dissipative model, in Eq. (A1), allows for an analytical solution that holds for any value of the field amplitudes. We now derive the equations of motion (EOM) in the presence of external driving fields $\varepsilon_{1,2}(t)$. From Eq. (A1), the time evolutions for the resonator operators $(x(t), p(t))$, in the Heisenberg picture, read

$$
\begin{aligned}
& \dot{x}(t)=\frac{p(t)}{m}-\varepsilon_{2}(t), \\
& \dot{p}(t)=-m \omega_{0}^{2} x(t)+\sum_{k=1}^{\infty} c_{k}\left(X_{k}-\frac{c_{k}}{m_{k} \omega_{k}^{2}} x(t)\right)+\varepsilon_{1}(t),
\end{aligned}
$$

similarly, the time evolutions for the oscillator operators $\left(X_{k}(t), P_{k}(t)\right)$ are

$$
\begin{aligned}
& \dot{X}_{k}(t)=\frac{P_{k}(t)}{m_{k}}, \\
& \dot{P}_{k}(t)=-m_{k} \omega_{k}^{2} X_{k}(t)+c_{k} x(t) .
\end{aligned}
$$

Solving the EOM for the bath degrees of freedom as a function of the operator $x(t)$ and substituting into Eq. (A5) one obtains the so-called generalized quantum Langevin equation [50]

$$
\begin{aligned}
& \ddot{x}(t)+\int_{t_{0}}^{+\infty} d t^{\prime} \gamma\left(t-t^{\prime}\right) \dot{x}\left(t^{\prime}\right)+\omega_{0}^{2} x(t) \\
& =-\gamma\left(t-t_{0}\right) x\left(t_{0}\right)+\frac{\xi(t)+\varepsilon_{1}(t)}{m}-\dot{\varepsilon}_{2}(t),
\end{aligned}
$$

with $t_{0} \rightarrow-\infty$ the initial time. It is worth to notice that this equation, derived starting from quantum operators, is equivalent to the classical Langevin equation. The function

$$
\gamma(t)=\frac{\theta(t)}{m} \sum_{k=1}^{\infty} \frac{c_{k}^{2}}{m_{k} \omega_{k}^{2}} \cos \left(\omega_{k} t\right)
$$

represents the memory damping kernel and

$$
\xi(t)=\sum_{k=1}^{\infty} c_{k}\left[X_{k}\left(t_{0}\right) \cos \omega_{k}\left(t-t_{0}\right)+\frac{P_{k}\left(t_{0}\right)}{m_{k} \omega_{k}} \sin \omega_{k}\left(t-t_{0}\right)\right],
$$

is the operator-valued fluctuating force which depends explicitly on the initial conditions of the bath position/momentum operators $X_{k}\left(t_{0}\right)$ and $P_{k}\left(t_{0}\right)$. Notice that the inhomogeneous term $\gamma\left(t-t_{0}\right) x\left(t_{0}\right)$ on the left-hand side (1.h.s.) of Eq. (A7) is a typical transient contribution and in our case, with $t_{0} \rightarrow$ $-\infty$, for times $t>0$ decays to zero.

In order to specify the reduced system dynamics it is now necessary to define the initial condition of the density matrix, which will also fix the statistical properties of the quantum noise. The usual choice, which allows to consider $\xi(t)$ as a stochastic force with zero average, is a factorized initial preparation $\rho_{\text {tot }}\left(t_{0}\right)=\rho_{S}\left(t_{0}\right) \otimes \rho_{R}\left(t_{0}\right)$ given by the product between the initial system density $\rho_{S}\left(t_{0}\right)$ and the density matrix of the bath alone, which is assumed in thermal equilibrium $\rho_{R}\left(t_{0}\right)=\exp \left(-H_{\mathrm{R}} / T\right) / \operatorname{Tr}\left\{\exp \left(-H_{\mathrm{R}} / T\right)\right\}$. With this choice we can evaluate the averages over the initial total density matrix of time dependent operators associated both to the bath and to the system. In the following, the notation $\langle A\rangle \equiv \operatorname{Tr}\left\{\rho_{\text {tot }}\left(t_{0}\right) A\right\}$ will represent the quantum average of a generic operator $A$. It is easy to see that $\langle\xi(t)\rangle=0$, and consequently from Eq. (A7) the following EOM for the average position $\langle x(t)\rangle\left(t_{0} \rightarrow-\infty\right)$

$$
\langle\ddot{x}(t)\rangle+\int_{-\infty}^{+\infty} d t^{\prime} \gamma\left(t-t^{\prime}\right)\left\langle\dot{x}\left(t^{\prime}\right)\right\rangle+\omega_{0}^{2}\langle x(t)\rangle=\frac{\varepsilon_{1}(t)}{m}-\dot{\varepsilon}_{2}(t) .
$$

The possible quantum features are eventually inside the properties of the reservoir treated as a quantum bath. Within the reduced description of the system alone, all quantities characterizing the environment are expressed in terms of the spectral density of the bath

$$
J(\omega)=\frac{\pi}{2} \sum_{k=1}^{\infty} \frac{c_{k}^{2}}{m_{k} \omega_{k}} \delta\left(\omega-\omega_{k}\right) .
$$


For example the damping kernel $\gamma(t)$ defined in Eq. (A8) can be written as

$$
\gamma(t)=\frac{2}{\pi m} \theta(t) \int_{0}^{\infty} d \omega \frac{J(\omega)}{\omega} \cos (\omega t) .
$$

Also the noise correlation function of the fluctuating force $\xi(t)$ becomes $\left\langle\xi(t) \xi\left(t^{\prime}\right)\right\rangle=\left\langle\xi\left(t-t^{\prime}\right) \xi(0)\right\rangle$ with [50]

$$
\langle\xi(t) \xi(0)\rangle=\hbar \int_{0}^{\infty} \frac{d \omega}{\pi} J(\omega)\left[\operatorname{coth}\left(\frac{\hbar \omega}{2 T}\right) \cos (\omega t)-i \sin (\omega t)\right] .
$$

In the last expression, we have reintroduced the $\hbar$ factor in order to emphasize the quantum character of the stochastic force correlators which in the classical case would corresponds to the limit $\hbar \rightarrow 0$, i.e., $\langle\xi(t) \xi(0)\rangle=m \gamma(t) T$.

To determine the time evolution of the system we now need to specify the frequency behavior of $J(\omega)$, and in particular its low frequency scaling, which determines the long time dynamics. We thus consider the realistic class of reservoirs with a power law behavior $J(\omega) \propto \omega^{s}$ at low frequency, here the index $s$ distinguishes between different dissipative influence: $s=1$ corresponds to an Ohmic bath, $s<1$ sub-Ohmic behavior and $s>1$ super-Ohmic behavior. In the following, we will focus on the range $0<s<2$. In order to describe a realistic heat bath, a cut off for the spectral density at high frequencies have to be considered. As done in the main text, we choose an algebraic Lorentzian cutoff, with the reservoir described in the continuum limit by

$$
J(\omega)=m \gamma_{\mathrm{s}} \bar{\omega}^{1-s} \frac{\omega^{s}}{1+\left(\omega / \omega_{\mathrm{c}}\right)^{2}},
$$

where $\gamma_{\mathrm{s}}$ is the friction amplitude, $\bar{\omega}$ is a characteristic frequency of the bath and $\omega_{c}$ is the largest frequency playing the role of the cutoff.

We recall that we are interested in the dynamics at times $t>0$. In this case, the transient homogeneous part, which depends on the initial condition averages $\langle x(-\infty)\rangle,\langle p(-\infty)\rangle$, will decay and vanish in the interval $(-\infty, 0)$. We are then left to find only the inhomogeneous solution driven by the external fields $\varepsilon_{1,2}$. This solution can be conveniently obtained transforming Eq. (A10) in the frequency domain. Defining the Fourier transform $f(\omega)=\int_{-\infty}^{+\infty} d t e^{i \omega t} f(t)$, we have

$$
\begin{aligned}
- & \omega^{2}\langle x(\omega)\rangle-i \omega \gamma(\omega)\langle x(\omega)\rangle+\omega_{0}^{2}\langle x(\omega)\rangle \\
= & \frac{\varepsilon_{1}(\omega)}{m}+i \omega \varepsilon_{2}(\omega) .
\end{aligned}
$$

From Eqs. (A15) and (A5), we obtain the exact solutions

$$
\begin{aligned}
& \langle x(\omega)\rangle=-\chi_{11}(\omega)\left[\varepsilon_{1}(\omega)+i m \omega \varepsilon_{2}(\omega)\right], \\
& \langle p(\omega)\rangle=i m \chi_{11}(\omega)\left[\varepsilon_{1}(\omega)+i m \omega \varepsilon_{2}(\omega)\right]+m \varepsilon_{2}(\omega),
\end{aligned}
$$

written in terms of the dynamical susceptibility

$$
\chi_{11}(\omega)=\frac{1}{m} \frac{1}{\omega^{2}-\omega_{0}^{2}+i \omega \gamma(\omega)} .
$$

In the time domain, this latter function corresponds to the retarded response function

$$
\chi_{11}\left(t-t^{\prime}\right)=-i \theta\left(t-t^{\prime}\right)\left\langle\left[x(t), x\left(t^{\prime}\right)\right]\right\rangle_{T},
$$

where the average $\langle\ldots\rangle_{T}$ is over the thermal equilibrium density matrix $\rho_{T}=\exp \left(-H_{0} / T\right) / \operatorname{Tr}\left\{\exp \left(-H_{0} / T\right)\right\}$, with $H_{0}$ the overall Hamiltonian in Eq.(A1), excluding the driving terms $\varepsilon_{1,2}(t)$, and the time evolution of $x(t)$ is with respect to $H_{0}$. The solutions (A16) can be rewritten in a more compact way introducing additional response functions $\chi_{i j}(\omega)$ defined as

$$
\begin{aligned}
& \chi_{12}(\omega)=i m \omega \chi_{11}(\omega), \\
& \chi_{21}(\omega)=-i m \omega \chi_{11}(\omega), \\
& \chi_{22}(\omega)=-m+m^{2} \omega^{2} \chi_{11}(\omega) .
\end{aligned}
$$

These correspond to all possible additional correlators with $x$ and $p$ operators, namely,

$$
\begin{aligned}
& \chi_{12}\left(t-t^{\prime}\right)=-i \theta\left(t-t^{\prime}\right)\left\langle\left[x(t), p\left(t^{\prime}\right)\right]\right\rangle_{T}, \\
& \chi_{21}\left(t-t^{\prime}\right)=-i \theta\left(t-t^{\prime}\right)\left\langle\left[p(t), x\left(t^{\prime}\right)\right]\right\rangle_{T}, \\
& \chi_{22}\left(t-t^{\prime}\right)=-i \theta\left(t-t^{\prime}\right)\left\langle\left[p(t), p\left(t^{\prime}\right)\right]\right\rangle_{T} .
\end{aligned}
$$

Using these correlators we can write the solutions as

$$
\begin{aligned}
& \langle x(\omega)\rangle=-\chi_{11}(\omega) \varepsilon_{1}(\omega)-\chi_{12}(\omega) \varepsilon_{2}(\omega), \\
& \langle p(\omega)\rangle=-\chi_{21}(\omega) \varepsilon_{1}(\omega)-\chi_{22}(\omega) \varepsilon_{2}(\omega) .
\end{aligned}
$$

Before closing this part, some comments are in order. (i) The linearity of the EOM (A10) implies an exact solution linear in the external fields $\varepsilon_{1,2}$ without higher order contributions, in addition the retarded correlators $\chi_{i, j}(\omega)$ do not depend on temperature and are equal to their classical counterparts.

(ii) As quoted in the main part, the solution in Eq. (A21) can be also expressed in the time domain as a convolution

$$
\begin{aligned}
& \langle x(t)\rangle=-\sum_{j=1}^{2} \int_{-\infty}^{+\infty} d t^{\prime} \chi_{1 j}\left(t-t^{\prime}\right) \varepsilon_{j}\left(t^{\prime}\right), \\
& \langle p(t)\rangle=-\sum_{j=1}^{2} \int_{-\infty}^{+\infty} d t^{\prime} \chi_{2 j}\left(t-t^{\prime}\right) \varepsilon_{j}\left(t^{\prime}\right) .
\end{aligned}
$$

(iii) The memory-friction kernel $\gamma\left(t-t^{\prime}\right)$, present in the Langevin equation, is completely determined via its Fourier transform $\gamma(\omega)$ which, using the Lorenzian shape of the spectral density in Eq. (A14), can be written as

$$
\gamma(\omega)=\frac{2 \gamma_{\mathrm{s}}}{\pi} \int_{0}^{\infty} d \omega_{1} \frac{\left(\omega_{1} / \bar{\omega}\right)^{s-1}}{1+\left(\omega_{1} / \omega_{c}\right)^{2}} \int_{0}^{\infty} d t \cos \left(\omega_{1} t\right) e^{i \omega t}
$$

Employing now the well-known identity $\int_{-\infty}^{+\infty} d x e^{-i \omega x} \theta(x)=\pi \delta(\omega)-i$ P.V. $(1 / \omega), \quad$ with the last term denoting the principal value, we obtain an explicit result for the real and imaginary parts of $\gamma(\omega)=\gamma^{\prime}(\omega)+i \gamma^{\prime \prime}(\omega)$

$$
\begin{aligned}
\gamma^{\prime}(\omega)= & \gamma_{s}\left|\frac{\omega}{\bar{\omega}}\right|^{s-1} \frac{1}{1+\left(\omega / \omega_{c}\right)^{2}}, \\
\gamma^{\prime \prime}(\omega)= & \frac{\gamma_{s} \operatorname{sgn} \omega}{1+\left(\omega / \omega_{c}\right)^{2}}\left|\frac{\omega}{\bar{\omega}}\right|^{s-1}[\cot (\pi s / 2) \\
& \left.+\left|\frac{\omega}{\omega_{c}}\right|^{2-s} \frac{1}{\sin (\pi s / 2)}\right] .
\end{aligned}
$$


Below we quote the behavior of $\gamma(\omega)$ in the realistic regime of interest $\omega \ll \omega_{c}$ which is used in the main text. We have

$$
\begin{aligned}
& \gamma^{\prime}(\omega)=\gamma_{\mathrm{s}}\left|\frac{\omega}{\bar{\omega}}\right|^{s-1}, \\
& \gamma^{\prime \prime}(\omega)=\gamma_{\mathrm{s}} \cot (\pi s / 2) \operatorname{sgn}(\omega)\left|\frac{\omega}{\bar{\omega}}\right|^{s-1},
\end{aligned}
$$

valid for $0<s<2$.

\section{APPENDIX B: AVERAGE POWERS AND THEIR FLUCTUATIONS}

The power contributions along the two different channels can be written as

$$
P_{1}(t)=-\dot{\varepsilon}_{1}(t)\langle x(t)\rangle, \quad P_{2}(t)=-\dot{\varepsilon}_{2}(t)\langle p(t)\rangle .
$$

Using Eq. (A22), the powers become

$$
P_{i}(t)=\dot{\varepsilon}_{i}(t) \sum_{j=1}^{2} \int_{-\infty}^{+\infty} d \tau \chi_{i j}(\tau) \varepsilon_{j}(t-\tau)
$$

Hereafter, we fix the fields $\left(\varepsilon_{1}(t), \varepsilon_{2}(t)\right)$, as done in the main text: we choose monochromatic functions with equal frequency $\omega$ with a phase shift $\varphi$

$$
\begin{aligned}
& \varepsilon_{1}(t)=\varepsilon_{1} \sin (\omega t), \\
& \varepsilon_{2}(t)=\varepsilon_{2} \cos (\omega t-\varphi) .
\end{aligned}
$$

With this choice it is easy to see that the powers $P_{i}(t)$ are periodic functions of time $t$, with period $\mathcal{T}=2 \pi / \omega$. We can thus compute their average over the period $\mathcal{T}$ defined as

$$
P_{i}=\frac{1}{\mathcal{T}} \int_{\bar{t}}^{\mathcal{T}+\bar{t}} d t P_{i}(t)
$$

with $\bar{t}$ a generic positive time. Notice that, due to the bilinear form in the external fields (B2), signals with different frequencies will not give rise to a finite average power. This explains why we fixed the same frequency $\omega$ in $\varepsilon_{1}$ and $\varepsilon_{2}$. By substituting Eq. (B3) into Eq. (B2) and averaging over $\mathcal{T}$, we obtain

$$
P_{i}=\varepsilon_{i} \sum_{j=1,2} \varepsilon_{j} \mathcal{L}_{i j}(\omega),
$$

where $\mathcal{L}_{i j}(\omega)$ are generalized Onsager coefficients. After straightforward manipulations, they can be written in terms of the retarded response functions (A17) as follows:

$$
\begin{aligned}
& \mathcal{L}_{11}(\omega)=-\frac{\omega}{2} \Im\left[\chi_{11}(\omega)\right] \\
& \mathcal{L}_{22}(\omega)=-\frac{m^{2} \omega^{3}}{2} \Im\left[\chi_{11}(\omega)\right] \\
& \mathcal{L}_{12}(\omega, \varphi)=-\frac{m \omega^{2}}{2}\left[\sin \varphi \Re\left[\chi_{11}(\omega)\right]+\cos \varphi \Im\left[\chi_{11}(\omega)\right]\right] \\
& \mathcal{L}_{21}(\omega, \varphi)=\mathcal{L}_{12}(\omega,-\varphi)
\end{aligned}
$$

Equations (B5) and (B6) allow us to compute the mean powers of the isothermal engine for given values of the driving frequency $\omega$, phase difference $\varphi$ and field amplitudes $\left(\varepsilon_{1}, \varepsilon_{2}\right)$.

Due to the presence of dissipation, the mean powers in Eq. (B5) undergo fluctuations, which would affect the engine performance. Fluctuations, during the whole time interval $t-t_{0}$, are described in terms of the power autocorrelation function. We thus introduce the deviation power operators

$$
\begin{aligned}
& \delta P_{1}(t)=-\dot{\varepsilon}_{1}(t)[x(t)-\langle x(t)\rangle], \\
& \delta P_{2}(t)=-\dot{\varepsilon}_{2}(t)[p(t)-\langle p(t)\rangle],
\end{aligned}
$$

in terms of which power fluctuations can be written as

$$
D_{i}(t)=\frac{1}{t-t_{0}} \int_{t_{0}}^{t} d t_{2} \int_{t_{0}}^{t} d t_{1}\left\langle\delta P_{i}\left(t_{2}\right) \delta P_{i}\left(t_{1}\right)\right\rangle,
$$

where $t-t_{0}$ is the total time interval with $t_{0} \rightarrow-\infty$. In this case one can perform the large interval limit $t-t_{0} \rightarrow \infty$ reducing the integrated autocorrelation function to a single integral

$$
D_{i}(t)=\int_{-\infty}^{t} d t_{1}\left[\left\langle\delta P_{i}(t) \delta P_{i}\left(t_{1}\right)\right\rangle+\left\langle\delta P_{i}\left(t_{1}\right) \delta P_{i}(t)\right\rangle\right] .
$$

Performing the change of variable $\tau=t-t_{1}$, one obtains

$$
D_{i}(t)=\int_{0}^{\infty} d \tau\left[\left\langle\delta P_{i}(t) \delta P_{i}(t-\tau)\right\rangle+\left\langle\delta P_{i}(t-\tau) \delta P_{i}(t)\right\rangle\right]
$$

For sake of brevity, in what follows we focus on $D_{1}(t)$, the equations for $i=2$ being analogous. Inserting the expression (B7) it follows:

$$
D_{1}(t)=\dot{\varepsilon}_{1}(t) \int_{0}^{\infty} d \tau \dot{\varepsilon}_{1}(t-\tau) C(t, t-\tau),
$$

where

$$
C\left(t, t^{\prime}\right)=\left\langle x(t) x\left(t^{\prime}\right)\right\rangle+\left\langle x\left(t^{\prime}\right) x(t)\right\rangle-2\langle x(t)\rangle\left\langle x\left(t^{\prime}\right)\right\rangle .
$$

We now demonstrate that from the linearity of Eq. (A7), the correlator $C\left(t, t^{\prime}\right)$ can be exactly computed and it does not depend on the external fields $\varepsilon_{1,2}$. Moreover, it reduces to a function of only the difference of time.

To evaluate Eq. (B12), we exploit the exact EOM of $x(t)$ in Eq. (A7), still at operatorial level, and passing in Fourier variables. We have

$$
x(\omega)=\langle x(\omega)\rangle-\chi_{11}(\omega) \xi(\omega) .
$$

Recalling that the fluctuating force has $\langle\xi\rangle=0$, we obtain

$$
\left\langle x(\omega) x\left(\omega^{\prime}\right)\right\rangle=\langle x(\omega)\rangle\left\langle x\left(\omega^{\prime}\right)\right\rangle+\chi_{11}(\omega) \chi_{11}\left(\omega^{\prime}\right)\left\langle\xi(\omega) \xi\left(\omega^{\prime}\right)\right\rangle .
$$

The last average is directly computed as Fourier transform of the noise correlator (A13). We have

$$
\left\langle\xi(\omega) \xi\left(\omega^{\prime}\right)\right\rangle=2 \pi m \omega \gamma^{\prime}(\omega)(1+\operatorname{coth}(\omega / 2 T)) \delta\left(\omega+\omega^{\prime}\right) .
$$

Inserting these results in the correlator (B12) one gets

$$
C\left(t, t^{\prime}\right)=C\left(t-t^{\prime}\right)=\int_{-\infty}^{\infty} \frac{d \omega}{2 \pi} e^{-i \omega\left(t-t^{\prime}\right)} C(\omega),
$$

with

$$
C(\omega)=-2 \Im\left[\chi_{11}(\omega)\right] \operatorname{coth}(\omega / 2 T),
$$

an even function of $\omega$. We are now in the position to determine the exact expression of the average power fluctuation 
(B11), $D_{1}=\frac{1}{\mathcal{T}} \int_{\bar{t}}^{\mathcal{T}+\bar{t}} d t D_{1}(t)$. We have

$$
D_{1}=\frac{\omega^{2} \varepsilon_{1}^{2}}{2} \int_{0}^{\infty} d \tau \cos (\omega \tau) C(\tau)=\frac{\omega^{2} \varepsilon_{1}^{2}}{4} C(\omega) .
$$

Inserting Eq. (B16) and using the expression (B6) for the Onsager coefficient $\mathcal{L}_{11}(\omega)$ it follows that

$$
D_{1}=\varepsilon_{1}^{2} \omega \operatorname{coth}(\omega / 2 T) \mathcal{L}_{11}(\omega) .
$$

It is worth to stress that the above result is exact and valid for any strength of $\varepsilon_{1}$, in other words it does not contain higher order contribution in $\varepsilon_{1}$. Analogous calculations for the fluctuation $D_{2}$ give the following result:

$$
D_{2}=\varepsilon_{2}^{2} \omega \operatorname{coth}(\omega / 2 T) \mathcal{L}_{22}(\omega) .
$$

Notice that, differently from the average powers in Eq. (B5), which take the same form in the quantum and classical setting, the power fluctuations substantially differ. In the high temperature regime, i.e., $T \gg \omega$, from Eq. (B20) the classical limit follows as

$$
D_{1}=\varepsilon_{1}^{2} 2 T \mathcal{L}_{11}(\omega), \quad D_{2}=\varepsilon_{2}^{2} 2 T \mathcal{L}_{22}(\omega) .
$$

\section{APPENDIX C: EXCESS POWER AND BATH POWER}

In this section, we show that the averaged excess power is dissipated into the bath. The equality holds true after averaging over one period of the driving fields, while in general the total excess power and the power drained by the bath show different time-dependent behaviours, related to the energy exchange between drives, system and reservoir. We recall the expressions for the powers related to each channel

$$
\begin{aligned}
& P_{1}(t)=-\dot{\varepsilon}_{1}(t)\langle x(t)\rangle, \\
& P_{2}(t)=-\dot{\varepsilon}_{2}(t)\langle p(t)\rangle,
\end{aligned}
$$

so that the excess power reads $P(t)=P_{1}(t)+P_{2}(t)$. For the sake of simplicity we restrict the following discussion to the relevant case of $\varphi=3 \pi / 2$, therefore the time-dependent drives are $\varepsilon_{1,2}(t)= \pm \varepsilon_{1,2} \sin (\omega t)$. From Eqs. (A22) and (A19), in the periodic steady state, $\langle x(t)\rangle$ and $\langle p(t)\rangle$ can be compactly written as

$$
\langle x(t)\rangle=x_{\omega} e^{i \omega t}+x_{\omega}^{*} e^{-i \omega t}
$$

and

$$
\langle p(t)\rangle=p_{\omega} e^{i \omega t}+p_{\omega}^{*} e^{-i \omega t},
$$

where the Fourier coefficients can be expressed in terms of the response function $\chi_{11}(\omega)$ as

$$
x_{\omega}=\frac{-m \omega \varepsilon_{2}+i \varepsilon_{1}}{2} \chi_{11}^{*}(\omega)
$$

and

$$
\begin{aligned}
p_{\omega} & =i m\left(\omega x_{\omega}+\varepsilon_{2} / 2\right) \\
& =\frac{1}{2}\left[-m \omega \varepsilon_{1} \chi_{11}^{*}(\omega)+i \varepsilon_{2}\left(m-m^{2} \omega^{2} \chi_{11}^{*}(\omega)\right)\right] .
\end{aligned}
$$

The instantaneous powers can be recast as follows:

$$
P_{i}(t)=P_{i}+P_{i, \omega} e^{2 i \omega t}+P_{i, \omega}^{*} e^{-2 i \omega t},
$$

with $i=1,2$ and the several terms reading

$$
\begin{aligned}
P_{1}= & -\omega \varepsilon_{1} \Re\left[x_{\omega}\right] \\
& =-\frac{\omega}{2}\left(\varepsilon_{1}^{2} \Im\left[\chi_{11}(\omega)\right]-m \omega \varepsilon_{1} \varepsilon_{2} \Re\left[\chi_{11}(\omega)\right]\right), \\
P_{2}= & \omega \varepsilon_{2} \Re\left[p_{\omega}\right]=-m \omega^{2} \varepsilon_{2} \Im\left[x_{\omega}\right] \\
= & -\frac{m \omega^{2}}{2}\left(\varepsilon_{1} \varepsilon_{2} \Re\left[\chi_{11}(\omega)\right]+m \omega \varepsilon_{2}^{2} \Im\left[\chi_{11}(\omega)\right]\right), \\
P_{1, \omega}= & -\frac{\omega \varepsilon_{1}}{2} x_{\omega}, \\
P_{2, \omega}= & \frac{\omega \varepsilon_{2}}{2} p_{\omega}=i \frac{m \omega}{2} \varepsilon_{2}\left(\omega x_{\omega}+\varepsilon_{2} / 2\right) .
\end{aligned}
$$

Taking the time average over one period $\mathcal{T}=2 \pi / \omega$ as

$$
F=\frac{1}{\mathcal{T}} \int_{0}^{\mathcal{T}} F(\tau) d \tau
$$

the excess power $P(t)=P_{1}(t)+P_{2}(t)$ averaged over one period becomes

$$
\begin{aligned}
P & =P_{1}+P_{2}=\omega\left(-\varepsilon_{1} \Re\left[x_{\omega}\right]+\varepsilon_{2} \Re\left[p_{\omega}\right]\right) \\
& =-\frac{\omega}{2} \Im\left[\chi_{11}(\omega)\right]\left(\varepsilon_{1}^{2}+m^{2} \omega^{2} \varepsilon_{2}^{2}\right) .
\end{aligned}
$$

We now consider the power associated to the reservoir, i.e., $\left\langle\dot{H}_{\mathrm{R}}(t)\right\rangle$ with $\langle\ldots\rangle$ denoting the quantum average. The aim is to demonstrate that the time average of this quantity, i.e., $\left\langle\dot{H}_{\mathrm{R}}\right\rangle$ coincides with the averaged excess power

$$
P=\left\langle\dot{H}_{\mathrm{R}}\right\rangle \text {. }
$$

Physically, this means that there is an energy exchange between input channel $P_{2}$, output channel $P_{1}$ and reservoir $\left\langle\dot{H}_{\mathrm{R}}\right\rangle$ : on average the energy is transferred from the input channel into the output one, with a fraction dissipated into the bath. Starting from the Hamiltonian of the reservoir in Eq. (A3), it is possible to derive the following expression:

$$
\begin{aligned}
\left\langle\dot{H}_{\mathrm{R}}(t)\right\rangle= & \frac{\langle\{x(t), \dot{\xi}(t)\}\rangle}{2} \\
& +\sum_{k=1}^{+\infty} \frac{c_{k}^{2}}{2 m_{k}} \int_{t_{0}}^{t} d s \cos \left(\omega_{k}(t-s)\right)\langle\{x(s), x(t)\}\rangle,
\end{aligned}
$$

where $\{a, b\}=a b+b a$ and $\xi(t)$, as written in Eq. (A9), represents the usual random force contribution with null mean value $\langle\xi(t)\rangle=0$. Expressing the second term in the above equation in terms of the bath correlation function $C\left(t, t^{\prime}\right)$ in Eq.(B12), after some cancellations one gets

$$
\left\langle\dot{H}_{\mathrm{R}}(t)\right\rangle=\sum_{k=1}^{+\infty} \frac{c_{k}^{2}}{m_{k}} \int_{t_{0}}^{t} d s \cos \left(\omega_{k}(t-s)\right)\langle x(s)\rangle\langle x(t)\rangle .
$$

Recalling $\gamma(t)=\theta(t) \bar{\gamma}(t)$ with

$$
\bar{\gamma}(t)=\frac{1}{m} \sum_{k=1}^{\infty} \frac{c_{k}^{2}}{m_{k} \omega_{k}^{2}} \cos \left(\omega_{k} t\right)
$$

and noticing that

$$
\frac{d}{d t} \int_{t_{0}}^{t} d s \dot{\bar{\gamma}}(t-s)\langle x(s)\rangle=\int_{t_{0}}^{t} d s \ddot{\bar{\gamma}}(t-s)\langle x(s)\rangle,
$$


having used that $\dot{\bar{\gamma}}(0)=0$. Eq. (C12) can thus be written as

$$
\left\langle\dot{H}_{\mathrm{R}}(t)\right\rangle=m\langle x(t)\rangle \frac{d}{d t} \int_{t_{0}}^{t} d s \frac{d \bar{\gamma}(t-s)}{d s}\langle x(s)\rangle .
$$

Integrating by parts one gets

$$
\begin{aligned}
\left\langle\dot{H}_{\mathrm{R}}(t)\right\rangle= & m\langle x(t)\rangle \frac{d}{d t}\left[\bar{\gamma}(0)\langle x(t)\rangle-\bar{\gamma}\left(t-t_{0}\right)\left\langle x\left(t_{0}\right)\right\rangle\right. \\
& \left.-\int_{t_{0}}^{t} d s \gamma(t-s)\langle\dot{x}(s)\rangle\right] .
\end{aligned}
$$

Employing the Langevin form of the EOM in Eq. (A10) and noting that the term proportional to $\left\langle x\left(t_{0}\right)\right\rangle$ vanishes for $t_{0} \rightarrow$ $-\infty$ and $\left\langle d^{3} x(t) / d t^{3}\right\rangle=-\omega^{2}\langle\dot{x}(t)\rangle$ we arrive at

$$
\left\langle\dot{H}_{\mathrm{R}}(t)\right\rangle=m\langle x(t)\rangle\left[\left(\bar{\gamma}(0)+\omega_{0}^{2}-\omega^{2}\right)\langle\dot{x}(t)\rangle-\frac{\dot{\varepsilon}_{1}(t)}{m}+\ddot{\varepsilon}_{2}(t)\right] .
$$

Finally, it is useful to rewrite the above expression as

$$
\begin{aligned}
\left\langle\dot{H}_{\mathrm{R}}(t)\right\rangle= & P(t)+m \frac{d}{d t}\left[\left(\bar{\gamma}(0)+\omega_{0}^{2}-\omega^{2}\right)\langle x(t)\rangle^{2}\right. \\
& \left.+\langle x(t)\rangle \dot{\varepsilon}_{2}(t)+\frac{\varepsilon_{2}^{2}(t)}{2}\right] .
\end{aligned}
$$

where we have recognized the excess power $P(t)$. Written in this form, it is easy to see that averaging over one period all the terms involved in the total derivative vanish, thus proving Eq. (C10). It is worth stressing that Eq. (C10) holds true only after taking the time average; indeed, time dependent excess energy contributions, and associated powers, are in general exchanged between different channels, i.e., system (S), the reservoir (R), and the interaction channel (SR). Averaging over one period, the only contribution that survives is proportional to the dissipation strength and it is related to the bath power $\left\langle\dot{H}_{\mathrm{R}}\right\rangle$, as just proved. Finally, as a representative example of the different dynamics inherited from the energy exchanges between drives, system and reservoir, in Fig. 4, we plot the input and output power [panel (a)], the excess power $P(t)$ and the bath power $\left\langle\dot{H}_{\mathrm{R}}(t)\right\rangle$ [panel (b)] as a function of time at a given frequency $\omega$ evaluated at the maximum efficiency ME (see the following section).

\section{APPENDIX D: FIGURES OF MERIT AT MAXIMUM EFFICIENCY}

Here we quote the expressions for the various figures of merit characterizing the isothermal engine, evaluated at the maximum efficiency (ME). Recalling the definition $\eta=$ $P_{\text {out }} / P_{\text {in }}$, and considering $P_{\text {out }}=-P_{1}$ with $P_{1}<0$ the power associated to the load, and $P_{\mathrm{in}}=P_{2}$ with $P_{2}>0$ the input power, the efficiency reads

$$
\eta=-\frac{\varepsilon_{1}^{2} \mathcal{L}_{11}+\varepsilon_{1} \varepsilon_{2} \mathcal{L}_{12}}{\varepsilon_{1} \varepsilon_{2} \mathcal{L}_{21}+\varepsilon_{2}^{2} \mathcal{L}_{22}},
$$

with Onsager coefficients reported in Eq. (B6). As stated in the main text, we chose the working point by maximizing the efficiency over $\varepsilon_{1}$, i.e., $\eta_{\mathrm{ME}}$ is defined by $\max _{\varepsilon_{1}}[\eta]$. Looking
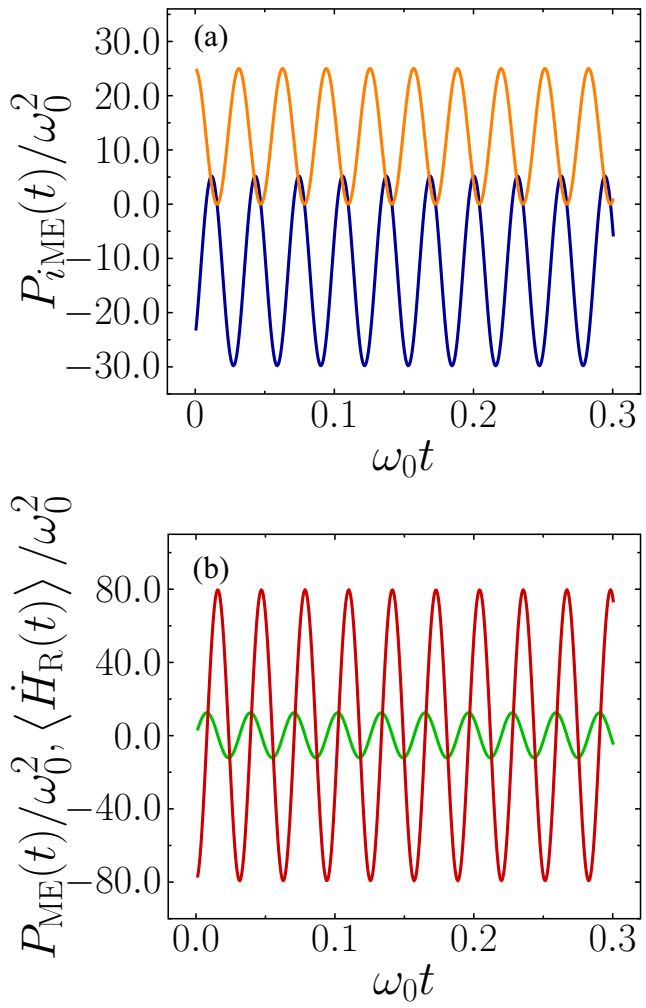

FIG. 4. (a) Input and output powers vs time at ME for $m \varepsilon_{2}^{2}=0.25 \omega_{0}, \omega=100 \omega_{0}, \bar{\omega}=\omega_{0}, \phi=3 \pi / 2, \gamma_{s}=0.8 \omega_{0}, s=$ 1 , and $\omega_{c}=4 \times 10^{4} \omega_{0}$ and (b) total excess power $P(t)$ (solid green curve) and power drained by the bath $\langle *\rangle \dot{H}_{\mathrm{R}}(t)$ (solid red curve) at ME.

for the maximum over $\varepsilon_{1}$, one gets [8]

$$
\varepsilon_{1, \mathrm{ME}}=\varepsilon_{2} \frac{\mathcal{L}_{22}}{\mathcal{L}_{21}}\left(\frac{1}{\sqrt{1+Y}}-1\right),
$$

where

$$
Y=\frac{\mathcal{L}_{12} \mathcal{L}_{21}}{\mathcal{L}_{11} \mathcal{L}_{22}-\mathcal{L}_{12} \mathcal{L}_{21}} .
$$

Inserting Eq. (D3) into Eq. (D1), one has

$$
\eta_{\mathrm{ME}}=X \frac{\sqrt{1+Y}-1}{\sqrt{1+Y}+1},
$$

where

$$
X=\frac{\mathcal{L}_{12}}{\mathcal{L}_{21}},
$$

is the asymmetry parameter. The corresponding average output power, at ME can be compactly written as

$$
P_{\mathrm{out}, \mathrm{ME}}=\varepsilon_{2}^{2} \eta_{\mathrm{ME}} \frac{\mathcal{L}_{22}}{\sqrt{1+Y}},
$$

with associated power fluctuation

$$
D_{\text {out }, \mathrm{ME}}=\varepsilon_{1, \mathrm{ME}}^{2} \omega \operatorname{coth}(\omega /(2 T)) \mathcal{L}_{11} .
$$




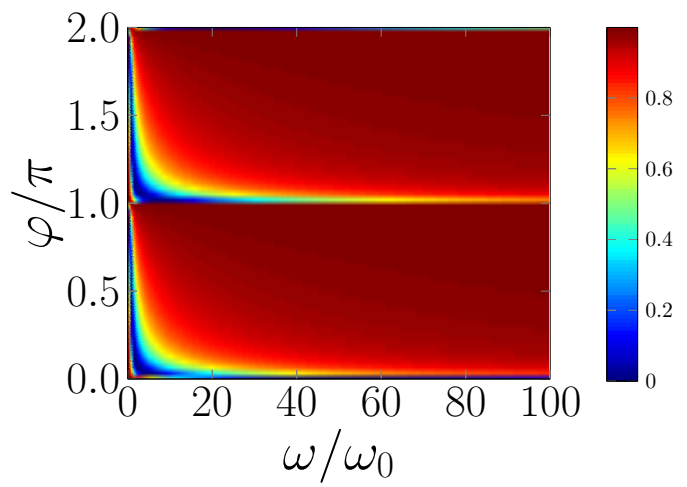

FIG. 5. Density plot of the maximum efficiency $\eta_{\mathrm{ME}}$ as function of $(\omega, \varphi)$ for $s=1, m \varepsilon_{2}^{2}=0.25 \omega_{0}, \gamma_{1}=0.5 \omega_{0}, \bar{\omega}=\omega_{0}$, and $\omega_{c}=$ $4 \times 10^{4} \omega_{0}$.

Note that the above expressions enter into the definition of the relative fluctuations, depicted in Fig. 2(c) in the main text

$$
\Sigma_{\mathrm{ME}}=\sqrt{\frac{D_{\mathrm{ou}, \mathrm{ME}}}{P_{\mathrm{out}, \mathrm{ME}}^{2}}} .
$$

\section{Power and efficiency with broken TRS}

The asymmetry parameter written in Eq. (D5) is related to the breaking of time reversal symmetry (TRS). Indeed, for $\varphi=0$ or $\pi$ one has $X=1$ (symmetric case), while other values of $\varphi$ would break TRS with $X \neq 1$. In the main text we have shown results fixing the phase factor $\varphi=3 \pi / 2$ where $X=-1$, i.e., the Onsager matrix is completely asymmetric. In the following we will focus the discussion by considering the limit of large cutoff frequency $\omega_{c} \gg \omega$. In Fig. 5, we report the density plot of the maximum efficiency $\eta_{\mathrm{ME}}$ as a function of $(\omega, \varphi)$, for $s=1$ and at fixed field amplitudes and dissipation strength. A similar plot is reported also in Fig. 6 for the output power at ME. We observe that at fixed driving frequency $\omega$ both quantities are periodic functions of the phase difference $\varphi$, with period $\pi$. In addition one can see that setting the engine operating regime at maximum asymmetry, i.e., $\varphi=k \pi / 2, k$ odd, an efficiency near to 1 with a finite optimal

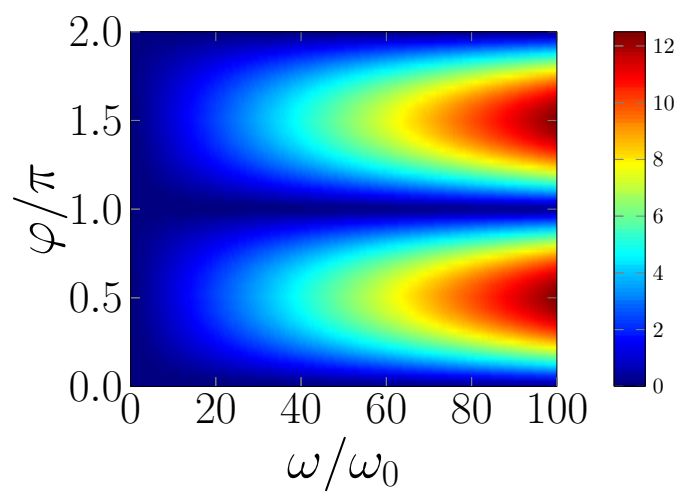

FIG. 6. Density plot of the output power at $\mathrm{ME} P_{\mathrm{out}, \mathrm{ME}} / \omega_{0}^{2}$ as function of $(\omega, \varphi)$ for $s=1, m \varepsilon_{2}^{2}=0.25 \omega_{0}, \gamma_{1}=0.5 \omega_{0}, \bar{\omega}=\omega_{0}$, and $\omega_{c}=4 \times 10^{4} \omega_{0}$. output power can be achieved in the antiadiabatic regime of high frequencies.

\section{Quasistatic and antiadiabatic limits}

It is useful to report the behavior of the quantities in Eq. (D4)-(D8), for $0<s<2$, in the two opposite frequency regimes: quasistatic limit $(\omega \rightarrow 0)$ and antiadiabatic (high frequencies, see below) regime. These asymptotic behaviours can be evaluated analytically for generic dissipation with power-law $s$, starting from the explicit expressions of the Onsager coefficients reported in Eq. (B6) and in the main text. We first discuss the low frequency regime. For sake of simplicity, we fix $\varphi=3 \pi / 2$. From Eq. (B6), the leading terms in the limit $\omega \rightarrow 0$ are

$$
\begin{aligned}
& \mathcal{L}_{11}(\omega) \simeq \frac{\bar{\gamma}_{s}}{2 m} \frac{\omega^{s+1}}{\omega_{0}^{4}}, \\
& \mathcal{L}_{12}(\omega)=-\mathcal{L}_{21}(\omega) \simeq-\frac{1}{2} \frac{\omega^{2}}{\omega_{0}^{2}}, \\
& \mathcal{L}_{22}(\omega) \simeq \frac{m \bar{\gamma}_{s}}{2} \frac{\omega^{s+3}}{\omega_{0}^{4}},
\end{aligned}
$$

with $\bar{\gamma}_{s}=\gamma_{s} \bar{\omega}^{1-s}$. For $\omega \rightarrow 0$, one obtains

$$
\begin{aligned}
& \eta_{\mathrm{ME}} \simeq 1-2 \frac{\gamma_{s} \bar{\omega}}{\omega_{0}^{2}}\left(\frac{\omega}{\bar{\omega}}\right)^{s}, \\
& P_{\mathrm{out}, \mathrm{ME}} \simeq \varepsilon_{2}^{2} \frac{m \omega^{3}}{2 \omega_{0}^{2}}, \\
& D_{\mathrm{out}, \mathrm{ME}} \simeq \frac{\gamma_{\mathrm{s}} m \varepsilon_{2}^{2} \bar{\omega}^{5}}{2 \omega_{0}^{4}} \operatorname{coth}\left(\frac{\omega}{2 T}\right)\left(\frac{\omega}{\bar{\omega}}\right)^{s+4}, \\
& \Sigma_{\mathrm{ME}} \simeq \sqrt{\frac{2 \gamma_{\mathrm{s}}}{m \varepsilon_{2}^{2} \bar{\omega}} \operatorname{coth}\left(\frac{\omega}{2 T}\right)\left(\frac{\omega}{\bar{\omega}}\right)^{s-2}} .
\end{aligned}
$$

Notice that, although in the quasistatic regime, the efficiency tends to the Carnot limit, the output power and the power fluctuations vanish, while the relative fluctuations diverge. The memory effects enter only the exponents of the different figures of merit. It follows that in the quasistatic regime energy conversion performance close to Carnot limit can be only achieved with vanishing output power, as recently discussed in related literature [70].

Let us consider now the opposite antiadiabatic regime at large frequencies defined by the condition $\tilde{\omega} \ll \omega$ with $\tilde{\omega}=\max \left\{\omega_{0}, \bar{\omega}\left(\gamma_{s} / \bar{\omega}\right)^{1 /(2-s)}\left[1+\cot ^{2}(\pi s / 2)\right]^{1 /(4-2 s)}\right\}$. In this regime, the following scaling for the figures of merit are obtained

$$
\begin{aligned}
& \eta_{\mathrm{ME}} \approx 1-2 \frac{\gamma_{\mathrm{s}}}{\bar{\omega}}\left(\frac{\omega}{\bar{\omega}}\right)^{s-2}, \\
& P_{\mathrm{out}, \mathrm{ME}} \approx \frac{\varepsilon_{2}^{2} m}{2} \omega, \\
& D_{\mathrm{out}, \mathrm{ME}} \approx \frac{m}{2} \varepsilon_{2}^{2} \gamma_{\mathrm{s}} \bar{\omega} \operatorname{coth}\left(\frac{\omega}{2 T}\right)\left(\frac{\omega}{\bar{\omega}}\right)^{s}, \\
& \Sigma_{\mathrm{ME}} \approx \sqrt{\frac{2 \gamma_{\mathrm{s}}}{m \varepsilon_{2}^{2} \bar{\omega}} \operatorname{coth}\left(\frac{\omega}{2 T}\right)\left(\frac{\omega}{\bar{\omega}}\right)^{s-2}} .
\end{aligned}
$$




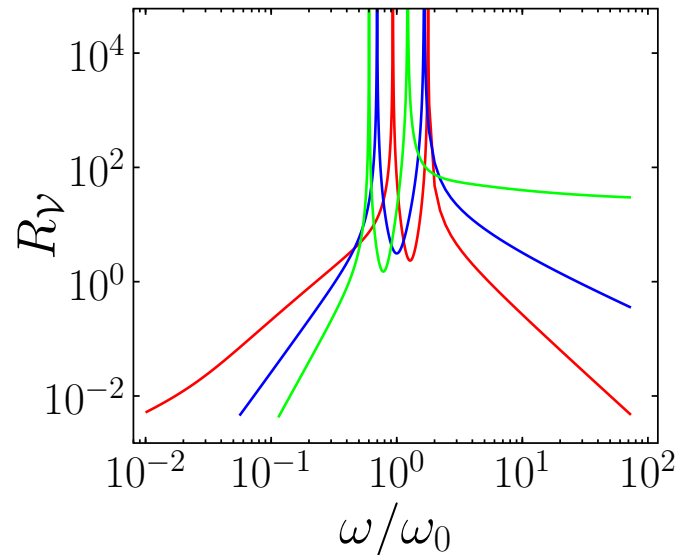

FIG. 7. Ratio of the trade-off parameter and the Seifert bound $\mathcal{V}$ at maximum efficiency plotted against the driving frequency $\omega$ for $s=\{0.5,1,1.5\}$ (red line, blue line, green line respectively). The model parameters have been set as follows: $m \varepsilon_{2}^{2}=0.25 \omega_{0}$, $\gamma_{\mathrm{s}}=0.5 \omega_{0}, \varphi=3 \pi / 2, T=0.01 \omega_{0}, \bar{\omega}=\omega_{0}$, and $\omega_{c}=4 \times 10^{4} \omega_{0}$.

From the above asymptotic scalings, one can recognize that also in the antiadiabatic regime the maximum efficiency tends to the Carnot limit $\eta_{\mathrm{ME}} \rightarrow 1$. However, while in the low frequency regime the output power vanishes $\propto \omega^{3}$, here a diverging power $\propto \omega$ is obtained. Furthermore, at high frequency, relative uncertainties tend to vanish, thus the isothermal engine shows remarkable performance. It is worth to note that memory effects, related to non-Ohmic dissipation, do not affect the behavior of the leading term in the output power. An explicit dependence on $s$ enters only into subleading contributions, which scale as $O\left(\omega^{s-1}\right)$.

\section{APPENDIX E: TRADE-OFF PARAMETER AND BOUNDS}

In the context of thermodynamic uncertainty relations (TURs) it is often introduced a so-called trade-off parameter

$$
\mathcal{Q}=\sigma \frac{D_{\text {out }}}{P_{\text {out }}^{2}}=\frac{1}{T}\left(P_{\text {in }}-P_{\text {out }}\right) \frac{D_{\text {out }}}{P_{\text {out }}^{2}},
$$

where $\sigma=\left(P_{\text {in }}-P_{\text {out }}\right) / T$ is the entropy production rate. This parameter quantifies the ability to achieve simultaneously good engine performance with finite output power and, possibly, low power fluctuations. As mentioned in the main text, several bounds have been introduced constraining the minimum value of the trade-off parameter. In particular, Koyuk and Seifert [46], considering a system under time-dependent drives, have derived the bound $\mathcal{Q} \geqslant \mathcal{V}$ with

$$
\mathcal{V}=2\left(1-\omega \frac{\partial_{\omega} P_{\text {out }}}{P_{\text {out }}}\right)^{2}
$$

Notice that this relation generalizes previous ones valid only for static driving [22], and holds true assuming overdamped and Markovian dynamics.

Considering the working point of the isothermal engine at maximum efficiency, the trade-off parameter can be written as

$$
\mathcal{Q}_{\mathrm{ME}}=\frac{1}{T}\left(\frac{1}{\eta_{\mathrm{ME}}}-1\right) \frac{D_{\mathrm{out}, \mathrm{ME}}}{P_{\mathrm{out}, \mathrm{ME}}} .
$$

In Fig. 7, we show the ratio of the trade-off parameter and the bound in Eq. (E2), $R_{\mathcal{V}}=(\mathcal{Q} / \mathcal{V})_{\mathrm{ME}}$, computed at $\mathrm{ME}$, for different kinds of dissipation, i.e., $s=0.5,1,1.5$. It is evident that the engine performance violates the bound in Eq. (E2), since several regions in the quasistatic and antiadiabatic regimes exist where the ratio $R_{\mathcal{V}}$ falls below 1 . Indeed, the violation is linked to the behavior of $\mathcal{Q}_{\mathrm{ME}}$ in the two opposite limits. At high frequencies, i.e., $\tilde{\omega}, T \ll \omega$ one gets the following asymptotic behaviors

$$
\mathcal{Q}_{\mathrm{ME}} \approx \frac{2 \gamma_{\mathrm{s}}^{2}}{T \bar{\omega}}\left(\frac{\omega}{\bar{\omega}}\right)^{2 s-3}
$$

and

$$
\mathcal{V}_{\mathrm{ME}} \approx 2 \text {, }
$$

which shows that the ratio $(\mathcal{Q} / \mathcal{V})_{\mathrm{ME}}$ tends to vanish for $0<$ $s<3 / 2$, while the bound is not violated for $s>3 / 2$.

Although in the two opposite regimes we can observe violation of this bound, in this case the asymptotic power law is
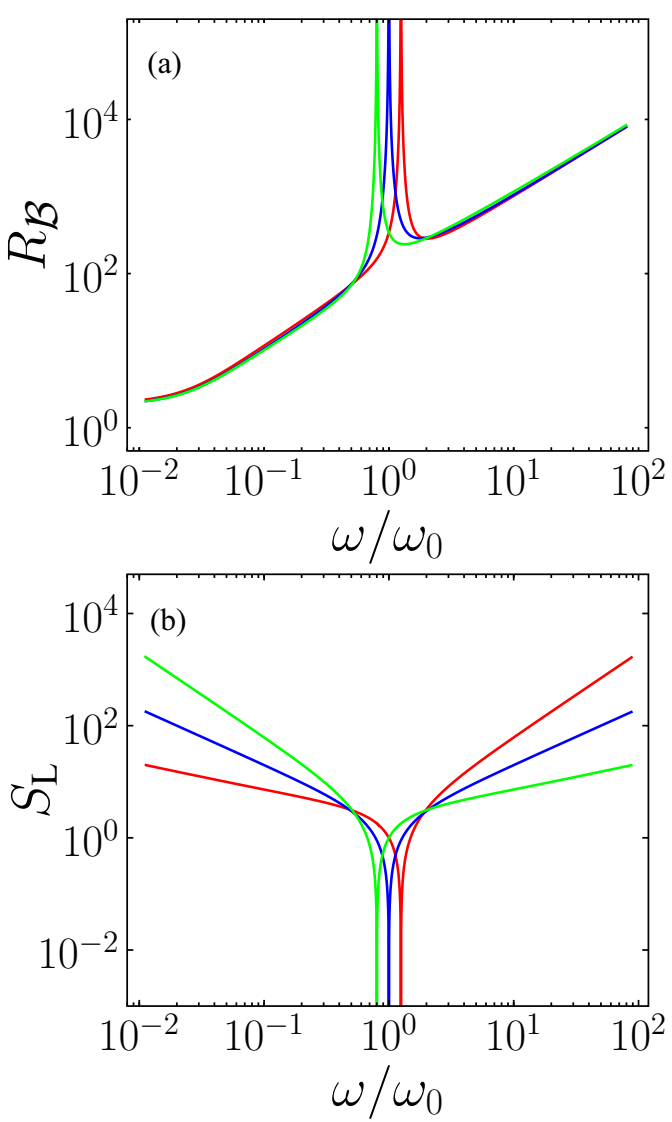

FIG. 8. Comparison of the engine performance against the unified TUR bound $\mathcal{B}$. In (a), the ratio $R_{\mathcal{B}}=(\mathcal{Q} / \mathcal{B})_{\mathrm{ME}}$ is plotted as function of the driving frequency for $s=\{0.5,1.0,1.5\}$ (red line, blue line, green line respectively). In (b), the asymmetry factor $S_{\mathrm{L}}$ at $\mathrm{ME}$ is plotted versus the driving frequency for the same values of the parameter $s$. In both panels, the model parameters have been set as follows: $m \varepsilon_{2}^{2}=0.25 \omega_{0}, \gamma_{\mathrm{s}}=0.5 \omega_{0}, T=0.01 \omega_{0}, \varphi=3 \pi / 2$, $\bar{\omega}=\omega_{0}$, and $\omega_{c}=4 \times 10^{4} \omega_{0}$. 
different. Indeed, for $\omega \rightarrow 0$ one has

$$
\mathcal{Q}_{\mathrm{ME}} \approx\left(\frac{2 \gamma_{\mathrm{s}}^{2} \bar{\omega}}{\omega_{0}^{2}}\right)^{2}\left(\frac{\omega}{\bar{\omega}}\right)^{2 s}
$$

and

$$
\mathcal{V}_{\mathrm{ME}} \approx 2
$$

It is found that the bound in Eq. (E2) is never obeyed in the quasistatic regime. We note that these possible violations in the quasi static regime are peculiar features of the model considered in this work. In particular, it is linked to the fact that one of the two time-dependent drives is coupled to the momentum $p$.

Indeed, from the associated equation of motion [see Eq. (A5)], we note that this model presents a fully underdamped dynamics in the whole frequency regime and, even in the quasistatic limit, it does not collapse to an overdamped motion for finite $\varepsilon_{2}$. Following these results, it is thus interesting to look for different formulations of the TUR bounds which could describe heat engine performance in the peculiar regime such as the one investigated here.

Under very general conditions, by making the assumption of validity of the linear response regime, Macieszczak et al. [63] have derived a unified TUR bound. The bound states that

$$
\mathcal{Q} \geqslant \mathcal{B}=\frac{2}{1+S_{\mathrm{L}}^{2}},
$$

where $S_{\mathrm{L}}$ indicates the asymmetry of the Onsager matrix [63] The model of isothermal engine under investigation, although not restricted to small values of the external driving strength, is written in terms of generalized Onsager coefficients and fulfill the bound posed by Eq. (E8) in the whole frequency range [see Fig. 8(a)].

Here, we report the frequency dependence of $S_{\mathrm{L}}$, that in the fully asymmetric case $\varphi=3 \pi / 2$ can be compactly written as [63]

$$
S_{\mathrm{L}}=\left|\mathcal{L}_{12}\right| / \sqrt{\mathcal{L}_{11} \mathcal{L}_{22}} .
$$

It is reported in Fig. 8(b), where in the two investigated frequency regions, one can notice a diverging behavior with frequency, occurring with different power-laws, for various $0<s<2$. This behavior agrees with the linear scaling, at high frequency, obtained for the ratio $R_{\mathcal{B}}=(\mathcal{Q} / \mathcal{B})_{\mathrm{ME}} \propto \omega$.

Finally, it is worth to report also the scaling behavior at high frequency of the entropy production at maximum efficiency, i.e., $\sigma_{\mathrm{ME}}$. From Eq. (D11), it reads

$$
\sigma_{\mathrm{ME}} \approx \frac{\varepsilon_{2}^{2} m \gamma_{s}}{T}\left|\frac{\omega}{\bar{\omega}}\right|^{s-1} \text {. }
$$

One can thus observe that, in the antiadiabatic regime, subOhmic bath $(s<1)$ results in a vanishing entropy production, while the latter saturates to a constant value in the Ohmic case $(s=1)$. Moreover, considering super-Ohmic bath $(1<$ $s<2$ ), the entropy production tends to grow with power law $\propto \omega^{s-1}$, whereas the squared relative power fluctuations keep on decreasing with power $\propto \omega^{s-2}$. As a consequence, the tradeoff parameter behaves as in Eq. (E4).
[1] M. Campisi, P. Hänggi, and P. Talkner, Colloquium: Quantum fluctuation relations: Foundations and applications, Rev. Mod. Phys. 83, 771 (2011).

[2] U. Seifert, Stochastic thermodynamics, fluctuation theorems and molecular machines, Rep. Prog. Phys. 75, 126001 (2012).

[3] R. Kosloff, Quantum thermodynamics: A dynamical viewpoint, Entropy 15, 2100 (2013).

[4] D. Gelbwaser-Klimovsky, W. Niedenzu, and G. Kurizki, Thermodynamics of quantum systems under dynamical control, Adv. At. Mol. Opt. Phys. 64, 329 (2015).

[5] B. Sothmann, R. Sánchez, and A. N. Jordan, Thermoelectric energy harvesting with quantum dots, Nanotechnology 26, 032001 (2015).

[6] J. Goold, M. Huber, A. Riera, L. del Rio, and P. Skrzypczyk, The role of quantum information in thermodynamics-a topical review, J. Phys. A: Math. Theor. 49, 143001 (2016).

[7] S. Vinjanampathy and J. Anders, Quantum thermodynamics, Contemp. Phys. 57, 545 (2016).

[8] G. Benenti, G. Casati, K. Saito, and R. S. Whitney, Fundamental aspects of steady-state conversion of heat to work at the nanoscale, Phys. Rep. 694, 1 (2017).

[9] R. S. Whitney, Most Efficient Quantum Thermoelectric at Finite Power Output, Phys. Rev. Lett. 112, 130601 (2014).

[10] W. Niedenzu, V. Mukherjee, A. Ghosh, A. G. Kofman, and G. Kurizki, Quantum engine efficiency bound beyond the second law of thermodynamics, Nat. Commun. 9, 165 (2018).
[11] N. Shiraishi and H. Tajima, Efficiency versus speed in quantum heat engines: Rigorous constraint from lieb-robinson bound, Phys. Rev. E 96, 022138 (2017).

[12] M. Campisi, J. Pekola, and R. Fazio, Nonequilibrium fluctuations in quantum heat engines: theory, example, and possible solid state experiments, New J. Phys. 17, 035012 (2015).

[13] F. Vischi, M. Carrega, P. Virtanen, E. Strambini, A. Braggio, and F. Giazotto, Thermodynamic cycles in Josephson junctions, Sci. Rep. 9, 3238 (2019).

[14] K. Ptaszyński, Coherence-enhanced constancy of a quantum thermoelectric generator, Phys. Rev. B 98, 085425 (2018).

[15] M. Bauer, K. Brandner, and U. Seifert, Optimal performance of periodically driven, stochastic heat engines under limited control, Phys. Rev. E 93, 042112 (2016).

[16] D. Gelbwaser-Klimovsky, W. Kopylov, and G. Schaller, Cooperative efficiency boost for quantum heat engines, Phys. Rev. A 99, 022129 (2019).

[17] K. Brandner and K. Saito, Thermodynamic Geometry of Microscopic Heat Engines, Phys. Rev. Lett. 124, 040602 (2020).

[18] P. Chattopadhyay, A. Mitra, and G. Paul, arXiv:1908.06804 [quant-ph].

[19] K. Ito, C. Jiang, and G. Watanabe, arXiv:1910.08096 [condmat.stat-mech].

[20] F. Carollo, F. M. Gambetta, K. Brandner, J. P. Garrahan, and I. Lesanovsky, Nonequilibrium Quantum Many-Body Rydberg Atom Engine, Phys. Rev. Lett. 124, 170602 (2020). 
[21] V. Holubec and A. Ryabov, Work and power fluctuations in a critical heat engine, Phys. Rev. E 96, 030102(R) (2017).

[22] P. Pietzonka and U. Seifert, Universal Trade-Off between Power, Efficiency, and Constancy in Steady-State Heat Engines, Phys. Rev. Lett. 120, 190602 (2018).

[23] G. Benenti, K. Saito, and G. Casati, Thermodynamic Bounds on Efficiency for Systems with Broken Time-Reversal Symmetry, Phys. Rev. Lett. 106, 230602 (2011).

[24] A. E. Allahverdyan, K. V. Hovhannisyan, A. V. Melkikh, and S. G. Gevorkian, Carnot Cycle at Finite Power: Attainability of Maximal Efficiency, Phys. Rev. Lett. 111, 050601 (2013).

[25] N. Shiraishi, Attainability of Carnot efficiency with autonomous engines, Phys. Rev. E 92, 050101(R) (2015).

[26] M. Campisi and R. Fazio, The power of a critical heat engine, Nat. Commun. 7, 11895 (2016).

[27] J. Konig and J. O. Indekeu, Engines with ideal efficiency and nonzero power for sublinear transport laws, Eur. Phys. J. B 89, 248 (2016).

[28] M. Polettini and M. Esposito, Carnot efficiency at divergent power output, Europhys. Lett. 118, 40003 (2017).

[29] J. S. Lee and H. Park, Carnot efficiency is reachable in an irreversible process, Sci. Rep. 7, 10725 (2017).

[30] V. Holubec and A. Ryabov, Diverging, but negligible power at Carnot efficiency: Theory and experiment, Phys. Rev. E 96, 062107 (2017).

[31] V. Holubec and A. Ryabov, Cycling Tames Power Fluctuations near Optimum Efficiency, Phys. Rev. Lett. 121, 120601 (2018).

[32] A. P. Solon and J. M. Horowitz, Phase Transition in Protocols Minimizing Work Fluctuations, Phys. Rev. Lett. 120, 180605 (2018).

[33] J. Uffink and J. H. van Lith-van Dis, arXiv:cond-mat/9806102 [cond-mat.stat-mech].

[34] A. C. Barato and U. Seifert, Thermodynamic Uncertainty Relation for Biomolecular Processes, Phys. Rev. Lett. 114, 158101 (2015).

[35] T. R. Gingrich, J. M. Horowitz, N. Perunov, and J. L. England, Dissipation Bounds All Steady-State Current Fluctuations, Phys. Rev. Lett. 116, 120601 (2016).

[36] U. Seifert, From stochastic thermodynamics to thermodynamic inference, Annu. Rev. Condens. Matter Phys. 10, 171 (2019).

[37] J. M. Horowitz and T. R. Gingrich, Thermodynamic uncertainty relations constrain non-equilibrium fluctuations, Nat. Phys. 16, 15 (2019).

[38] K. Brandner, T. Hanazato, and K. Saito, Thermodynamic Bounds on Precision in Ballistic Multiterminal Transport, Phys. Rev. Lett. 120, 090601 (2018).

[39] B. K. Agarwalla and D. Segal, Assessing the validity of the thermodynamic uncertainty relation in quantum systems, Phys. Rev. B 98, 155438 (2018).

[40] J. Liu and D. Segal, Thermodynamic uncertainty relation in quantum thermoelectric junctions, Phys. Rev. E 99, 062141 (2019).

[41] A. M. Timpanaro, G. Guarnieri, J. Goold, and G. T. Landi, Thermodynamic Uncertainty Relations from Exchange Fluctuation Theorems, Phys. Rev. Lett. 123, 090604 (2019).

[42] Y. Hasegawa and T. Van Vu, Fluctuation Theorem Uncertainty Relation, Phys. Rev. Lett. 123, 110602 (2019).

[43] K. Proesmans and J. M. Horowitz, Hysteretic thermodynamic uncertainty relation for systems with broken time-reversal symmetry, J. Stat. Mech. (2019) 054005.
[44] P. P. Potts and P. Samuelsson, Thermodynamic uncertainty relations including measurement and feedback, Phys. Rev. E 100, 052137 (2019).

[45] T. Van Vu and Y. Hasegawa, Thermodynamic uncertainty relations under arbitrary control protocols, Phys. Rev. Research 2, 013060 (2020).

[46] T. Koyuk and U. Seifert, Operationally Accessible Bounds on Fluctuations and Entropy Production in Periodically Driven Systems, Phys. Rev. Lett. 122, 230601 (2019).

[47] S. Kheradsoud, N. Dashti, M. Misiorny, P. P. Potts, J. Splettstoesser, and P. Samuelsson, Power, efficiency and fluctuations in a quantum point contact as steady-state thermoelectric heat engine, Entropy 21, 777 (2019).

[48] G. Falasco, E. M., and J. Delvenne, Unifying thermodynamic uncertainty relations, New J. Phys. 22, 053046 (2020).

[49] A. O. Caldeira and A. J. Leggett, Quantum tunneling in a dissipative system, Ann. Phys. 149, 374 (1983).

[50] U. Weiss, Quantum Dissipative Systems (World Scientific, Singapore, 2012).

[51] K. Proesmans, Y. Dreher, M. Gavrilov, J. Bechhoefer, and C. Van den Broeck, Brownian Duet: A Novel Tale of Thermodynamic Efficiency, Phys. Rev. X 6, 041010 (2016).

[52] K. Proesmans and C. Van den Broeck, The underdamped brownian duet and stochastic linear irreversible thermodynamics, Chaos 27, 104601 (2017).

[53] M. Carrega, M. Sassetti, and U. Weiss, Optimal work-to-work conversion of a nonlinear quantum brownian duet, Phys. Rev. A 99, 062111 (2019).

[54] L. M. Cangemi, V. Cataudella, G. Benenti, M. Sassetti, and G. De Filippis, Violation of thermodynamics uncertainty relations in a periodically driven work-to-work converter from weak to strong dissipation, Phys. Rev. B 102, 165418 (2020).

[55] G. Ingold and Y. V. Nazarov, Charge tunneling rates in ultrasmall junctions, in Single Charge Tunneling: Coulomb Blockade Phenomena In Nanostructures, edited by H. Grabert and M. H. Devoret (Springer US, Boston, MA, 1992), pp. 21-107.

[56] M. H. Devoret, Quantum fluctuations in electrical circuits, in Quantum Fluctuations Conference, Les Houches (France), Session LXIII, edited by S. Reynaud, E. Giacobino, and J. ZinnJustin, Les Houches (Elsevier, 1997).

[57] S. M. Girvin, Circuit QED: Superconducting Qubits Coupled to Microwave Photons (Oxford Scholarship, 2014).

[58] N. Freitas, J. C. Delvenne, and M. Esposito, Stochastic and Quantum Thermodynamics of Driven RLC Networks, Phys. Rev. X 10, 031005 (2020).

[59] P. Krantz, M. Kjaergaard, F. Yan, T. P. Orlando, S. Gustavsson, and W. D. Oliver, A quantum engineer's guide to superconducting qubits, Appl. Phys. Rev. 6, 021318 (2019).

[60] $\langle\ldots\rangle_{T}$ denotes averaging over the thermal equilibrium state $\rho_{T}=\exp \left(-H_{0} / T\right)$, where $H_{0}$ is the overall Hamiltonian, excluding the driving terms, with $x$ and $p$ evolving with respect to $H_{0}$.

[61] M. Wiedmann, J. T. Stockburger, and J. Ankerhold, Nonmarkovian dynamics of a quantum heat engine: out-ofequilibrium operation and thermal coupling control, New J. Phys. 22, 033007 (2020).

[62] If $P_{1}>0, P_{2}<0$ then $P_{\text {out }}=-P_{2}, P_{\text {in }}=P_{1}$. 
[63] K. Macieszczak, K. Brandner, and J. P. Garrahan, Unified Thermodynamic Uncertainty Relations in Linear Response, Phys. Rev. Lett. 121, 130601 (2018).

[64] K. Brandner, K. Saito, and U. Seifert, Thermodynamics of Micro- and Nano-Systems Driven by Periodic Temperature Variations, Phys. Rev. X 5, 031019 (2015).

[65] J. Jaramillo, M. Beau, and A. del Campo, Quantum supremacy of many-particle thermal machines, New J. Phys. 18, 075019 (2016).

[66] M. Ponmurugan, Efficiency at the maximum power of the power law dissipative Carnot-like heat engines with non-adiabatic dissipation, Commun. Theor. Phys. 72, 025601 (2020).
[67] H. J. D. Miller, M. H. Mohammady, M. Perarnau-Llobet, and G. Guarnieri, arXiv:2006.07316 [quant-ph].

[68] B. Bhandari, P. Terrén Alonso, F. Taddei, F. von Oppen, R. Fazio, and L. Arrachea, Geometric properties of adiabatic quantum thermal machines, Phys. Rev. B 102, 155407 (2020).

[69] S. S. Kadijani, T. L. Schmidt, M. Esposito, and N. Freitas, Heat transport in overdamped quantum systems, Phys. Rev. B 102, 235422 (2020).

[70] N. Shiraishi, K. Saito, and H. Tasaki, Universal Trade-Off Relation between Power and Efficiency for Heat Engines, Phys. Rev. Lett. 117, 190601 (2016). 